
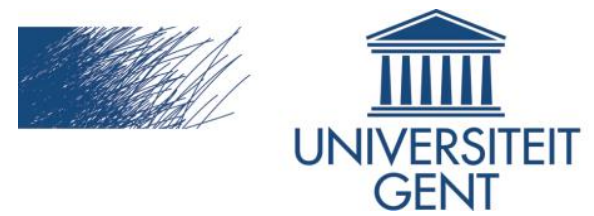

biblio.ugent.be

The UGent Institutional Repository is the electronic archiving and dissemination platform for all UGent research publications. Ghent University has implemented a mandate stipulating that all academic publications of UGent researchers should be deposited and archived in this repository. Except for items where current copyright restrictions apply, these papers are available in Open Access.

This item is the archived peer-reviewed author-version of: Synergy between intraperitoneal aerosolization (PIPAC) and cancer nanomedicine: cisplatin-loaded polyarginine-hyaluronicacid nanocarriers efficiently eradicate peritoneal metastasis of advanced human ovarian cancer

Authors: Shariati M., Lollo G., Matha K., Descamps B., Vanhove C., Van de Sande L., Willaert, W., Balcaen L., Vanhaecke F., Benoit J.P., Ceelen W., De Smedt S.C., Remaut K.

In: ACS Applied Materials \& Interfaces, 12(26): 29024-29036

To refer to or to cite this work, please use the citation to the published version:

Shariati M., Lollo G., Matha K., Descamps B., Vanhove C., Van de Sande L., Willaert, W., Balcaen L., Vanhaecke F., Benoit J.P., Ceelen W., De Smedt S.C., Remaut K. (2020) Synergy between intraperitoneal aerosolization (PIPAC) and cancer nanomedicine: cisplatin-loaded polyarginine-hyaluronic-acid nanocarriers efficiently eradicate peritoneal metastasis of advanced human ovarian cancer

ACS Applied Materials \& Interfaces, 12(26): 29024-29036

DOI: 10.1021/acsami.0c05554 


\title{
Synergy Between Intraperitoneal Aerosolization (PIPAC) and Cancer Nanomedicine: Cisplatin-Loaded Polyarginine-Hyaluronic Acid Nanocarriers Efficiently Eradicate Peritoneal Metastasis of Advanced Human Ovarian Cancer
}

\author{
Molood Shariati ${ }^{1,2}$, Giovanna Lollo ${ }^{3}$, Kevin Matha $^{4,5}$, Benedicte Descamps ${ }^{6}$, Christian Vanhove ${ }^{2,6}$, Leen \\ Van de Sande ${ }^{2,7}$, Wouter Willaert ${ }^{2,7}$, Lieve Balcaen ${ }^{8}$, Frank Vanhaecke ${ }^{8}$, Jean-Pierre Benoit ${ }^{4,5}$, Wim \\ Ceelen ${ }^{2,7}$, Stefaan C. De Smedt ${ }^{1,2^{*}}$, Katrien Remaut ${ }^{1,2^{*}}$
}

${ }^{1}$ Laboratory of General Biochemistry and Physical Pharmacy, Faculty of Pharmaceutical Sciences, Ghent University, 9000 Ghent, Belgium;

${ }^{2}$ Cancer Research Institute Ghent (CRIG), 9000 Ghent, Belgium;

${ }^{3}$ Univ Lyon, Université Lyon 1, CNRS, UMR5007, Laboratoire d'Automatique, de Génie des Procédés et de Génie Pharmaceutique (LAGEPP), 43 bd du 11 Novembre 1918, F-69622 Lyon, France;

${ }^{4}$ Micro et Nanomédecines Translationnelles, MINT, UNIV Angers, UMR INSERM 1066, UMR CNRS 6021, Angers, France ;

${ }^{5}$ CHU Angers, département Pharmacie, 4 rue Larrey, 49933 Angers cedex 9;

${ }^{6}$ Department of Electronics and Information Systems, Faculty of Engineering and Architecture, Ghent University, 9000 Ghent, Belgium;

${ }^{7}$ Department of GI Surgery, Ghent University Hospital and Laboratory for Experimental Surgery, Ghent University, Corneel Heymanslaan 10, 9000 Ghent, Belgium;

${ }^{8}$ Department of Analytical Chemistry, Atomic \& Mass Spectrometry-A\&MS Research Unit, Campus Sterre, Ghent University, Krijgslaan 281-S12, 9000 Ghent, Belgium.

E-mail: Katrien.Remaut@UGent.be; Stefaan.Desmedt@UGent.be

Tel: +32926480 76, Fax: +3292648189

*Corresponding address: Ottergemsesteenweg 460, 9000 Gent, Belgium

\begin{abstract}
Intra-abdominal dissemination of peritoneal nodules, a condition known as peritoneal carcinomatosis (PC), is typically diagnosed in ovarian cancer patients at the advanced stages. Current treatment of PC consists of perioperative systemic chemotherapy and cytoreductive surgery, followed by intraabdominal flushing with solutions of chemotherapeutics such as cisplatin and oxaliplatin. In this study, we developed cisplatin-loaded polyarginine-hyaluronic acid nanoscale particles (Cis-pARG-HA NPs) with high colloidal stability, marked drug loading efficiency, unimpaired biological activity and tumor targeting ability. Injected Cis-pARG-HA NPs showed enhanced antitumor activity in a rat model of PC, when compared to injection of the free cisplatin drug. The activity of Cis-pARG-HA NPs could even be further improved when administered by an intra-abdominal aerosol therapy, referred to as pressurized intraperitoneal aerosol chemotherapy (PIPAC). PIPAC is hypothesized to ensure a more homogeneous drug distribution together with a deeper drug penetration into peritoneal tumor nodules within the abdominal cavity. Using fluorescent pARG-HA NPs, this enhanced nanoparticle deposit on tumors could indeed be observed in regions opposite the aerosolization nozzle. Therefore, this study demonstrates that nanoparticles carrying chemotherapeutics can be synergistically combined with the PIPAC technique for IP therapy of disseminated advanced ovarian tumors, while this synergistic effect was not observed for the administration of free cisplatin.
\end{abstract}


Keywords: Nanomedicine, Cisplatin, Hyaluronic acid, Polyarginine, Peritoneal carcinomatosis, PIPAC, Aerosolization, Intraperitoneal drug delivery

\section{Introduction}

Ovarian cancer is one of the most commonly diagnosed cancers in women and is a leading cause of death among gynecological malignancies around the globe ${ }^{1}$. The high mortality rate associated with ovarian cancer is largely by the fact that this cancer has proven refractory to most existing therapeutic modalities owing to its well-known metastatic behavior. At the advanced stages (stages III and IV), ovarian cancer is characterized by the formation of disseminated tumor nodules on the peritoneal membrane, a condition referred to as peritoneal carcinomatosis (PC $)^{2}$. Thus far, the standard of care for patients diagnosed with PC involves surgical removal of all macroscopic peritoneal metastases (cytoreductive surgery) together with perioperative and/or postoperative systemic intravenous (IV) chemotherapy with platinum and taxane-based compounds ${ }^{3}$. The large surface area of the peritoneal cavity and poor vascularization of the peritoneum present, however, major obstacles to the effective eradication of PC via the current systemic chemotherapy regimens ${ }^{4}$. Therefore, local intraperitoneal (IP) administration of chemotherapeutics was introduced around forty years ago, to provide a sufficient therapeutic concentration in the peritoneal cavity in order to enhance the local therapeutic effect while minimizing systemic adverse effects ${ }^{5-7}$. Cytoreductive surgery (CRS), for example, is often combined with hyperthermic intraperitoneal chemotherapy (HIPEC), where the peritoneal cavity is flushed with a solution of chemotherapeutics at $38-42{ }^{\circ} \mathrm{C}$ for 30 minutes to several hours ${ }^{8-9}$. However, only selected patients with PC are eligible for CRS along with HIPEC based on their tumor type and the extent of disease ${ }^{10}$. For patients with unresectable tumors, a new minimally invasive, intra-abdominal aerosolbased localized therapy has emerged, known as pressurized intraperitoneal aerosol chemotherapy (PIPAC) ${ }^{4}$. In PIPAC, which is regarded as a palliative therapeutic option for patients suffering from PC, anticancer therapeutic agents are delivered into the closed and inflated peritoneal cavity as a pressurized normothermic aerosol, during a short laparoscopic procedure. It is speculated that the pressurized chemo-aerosols behave "gas-like", ensuring a homogeneous drug distribution throughout the entire peritoneal space ${ }^{4,11-12}$. Furthermore, compared to conventional IP lavage, it appears that the generated artificial pressure gradient, in the shape of a $12 \mathrm{mmHg} \mathrm{CO}_{2}$ pneumoperitoneum, counteracts the elevated intra-tumoral interstitial fluid pressure, thereby enhancing the penetration of chemotherapeutic drugs into the peritoneal nodules ${ }^{13}$. Nevertheless, the efficacy of localized IP chemotherapy is still hampered by pharmacological limitations such as rapid clearance of chemotherapeutics from the peritoneal cavity and minimal specificity towards cancer cells ${ }^{14}$. Therefore, most patients develop disease recurrence in a short time after the local administration of chemotherapeutics.

To prolong the residence time of chemotherapeutics in the peritoneal cavity, the encapsulation of chemotherapeutics into nanoparticles or controlled release systems is being evaluated. Furthermore, by applying surface modifications, nanoparticles could allow for optimal tumor targeting after administration in the peritoneal cavity ${ }^{15-17}$. A growing body of evidence has shown the potential of hyaluronic acid (HA), an anionic natural non-toxic mucoadhesive polysaccharide, for various biomedical and pharmaceutical applications (e.g., drug delivery, tissue engineering, cancer nanomedicine and molecular imaging) ${ }^{18-20}$. In the area of cancer nanotherapy, there has been a special emphasis on the role of HA as a ligand for cluster determinant 44 (CD44) ${ }^{21-22}$, which is considerably overexpressed on the surface of primary and metastatic peritoneal tumors ${ }^{23}$. Therefore, the specific binding of HA to the CD44 receptors could lead to preferential internalization of nanoparticles into cancer cells ${ }^{24-25}$. In addition, polyarginine ( $\mathrm{pARG}$ ), a cationic polyaminoacid, has been extensively utilized in drug delivery platforms due to its physicochemical and biopharmaceutical properties, biodegradability as well as good safety profile ${ }^{26-27}$. In this study, we have developed a cross-linked nanoparticle system (NP) composed of pARG and HA (pARG-HA NP) for the delivery of cisplatin, a 
platinum-based anticancer drug. We first investigated the physicochemical properties of pARG-HA NPs, the cisplatin encapsulation efficiency and the in vitro release kinetics. Then, we explored the in vitro colloidal stability and biological activity of pARG-HA NPs in undiluted human biofluids, such as ascites and assessed the in vivo antitumor efficacy of cisplatin-loaded pARG-HA NPs (Cis-pARG-HA NPs) in a rat model of peritoneally disseminated human ovarian cancer, compared to free cisplatin. Given the limited amount of evidence considering the advantage of administering chemotherapeutics or nanoparticles as an aerosol, compared to injection into the peritoneal cavity as a solution, IP delivery was performed both by injection and nebulization, using the clinically applied PIPAC procedure. Finally, tumor accumulation and in-tissue penetration depth of IP nebulized and injected pARG-HA NPs were evaluated using fluorescence confocal microscopy. In summary, this study aimed to create insights into the pros and cons of the administration technique (liquid injection versus nebulization), and the added value of a nanoparticulate delivery platform for the delivery of cisplatin into the peritoneal cavity.

\section{Results and Discussion}

\section{1. pARG and HA assemble into negatively charged nanoscale particles, displaying a long-term} stability

We developed a cross-linked nanoscale delivery vehicle consisting of two bioinspired and oppositely charged polymers, namely hyaluronic acid (HA) and polyarginine (pARG). Briefly, pARG-HA NPs were formed as a result of electrostatic complexation between HA and pARG at a concentration of 9 $\mathrm{mg} / \mathrm{mL}$ and $2.5 \mathrm{mg} / \mathrm{mL}$ (HA/pARG mass ratio: 4.5 ), respectively, at room temperature by the ionic gelation method ${ }^{27-29}$. The positively charged primary amines of pARG interact with the negatively charged carboxylic groups of the HA backbone, leading to the formation of a spherical polymeric nanocarrier (Figure 1A). The developed nanoformulation featured an average hydrodynamic diameter of $249 \pm 4 \mathrm{~nm}$ and a monodisperse size distribution (polydispersity index (PDI): $0.19 \pm 0.02$ ) in water as indicated by Dynamic Light Scattering (DLS) analysis. The deprotonated carboxyl groups in the HA polymer introduce a negative charge onto the surface of the NPs (zeta potential: $-24 \pm 1 \mathrm{mV}$ ) which is attributed to excess of HA relative to that of pARG in the formulation. Figure 1B depicts the stability profile of pARG-HA NPs over time. Evidently, pARG-HA NPs displayed a constant hydrodynamic diameter and surface charge in aqueous solution after isolation by centrifugation over the course of 84 days when stored at $4{ }^{\circ} \mathrm{C}$, suggesting a good physicochemical stability during long-term storage which is regarded as a crucial factor for adequate handling of the nanoformulations. In addition, it is inferred that the negatively charged surface of pARG-HA NPs may prevent the aggregation of nanoparticles through electrostatic repulsion and also provide long-term stability in aqueous solution.

\section{2. pARG-HA NPs efficiently encapsulate cisplatin and provide a sustained drug release over 8 days}

Cisplatin is a potent platinum-based anticancer drug, approved as a chemotherapeutic agent in $1978{ }^{30}$ ${ }^{31}$. Although it is now commonly used in medical oncology ${ }^{32}$, the clinical implementation still suffers from intrinsic and acquired drug resistance and severe adverse effects, such as acute nephrotoxicity and chronic neurotoxicity ${ }^{33-34}$. Encapsulation of platinum-based chemotherapeutic agents into nanoparticles can reduce off-target systemic toxicities and offer controlled drug release and protection of platinum drugs from degradation, thereby enhancing therapeutic efficacy ${ }^{35-36}$. For polymers containing ionic blocks, cisplatin can be incorporated through coordination binding with the polyion blocks in an aqueous medium. For a polycarboxylate like HA, the carboxylic moieties have the ability to substitute the chloride groups (as the anionic ligands) in cisplatin, thereby forming a metal coordination bond between the platinum (Pt) II and the carboxyl group in HA polymers (Figure 1A). In turn, the presence of 
abundant chloride ions can reverse this metal ion coordination bond, thereby regenerating free drug in chloride-rich environments ${ }^{33,37}$. To form a nanoparticle structure, the negatively charged HA can be mixed with positively charged pARG that acts as a cross-linking agent ${ }^{27,29}$.

Guided by these principles, cisplatin-loaded pARG-HA NPs (Cis-pARG-HA NPs) were synthetized by mixing an aqueous cisplatin solution at a concentration of $1 \mathrm{mg} / \mathrm{mL}$ with pARG and HA solutions at room temperature. To prevent the release of cisplatin by chloride ions, pARG-OH was used, obtained by hydroxylation of pARG-Cl. Cis-pARG-HA NPs displayed a slight decrease in size $(213 \pm 3 \mathrm{~nm})$ compared to blank pARG-HA NPs, reflecting the formation of a more compact nanostructure in the presence of cisplatin ${ }^{27,29}$. Drug loading efficiency and encapsulation efficiency were determined to be $8.1 \pm 0.01 \%(\mathrm{w} / \mathrm{w})$ and $74.7 \pm 0.1 \%(\mathrm{w} / \mathrm{w})$, respectively, as analyzed by inductively coupled plasmamass spectrometry (ICP-MS). The drug release kinetics of cisplatin from pARG-HA NPs exhibited two phases (Figure 1C). In the initial phase, an accelerated release profile was found with around $40 \%$ of total loaded cisplatin released from the nanosystem after 24 hours, followed by a sustained and slow release of drug over 8 days. This initial burst release may be ascribed to the release of cisplatin that is mainly complexed with the HA polymer on the surface of nanoparticles ${ }^{38-39}$, while the sustained release of cisplatin might occur via an inverse ligand exchange reaction of the Pt (II) atom from the carboxyl group in the polymer chains to chloride in the chloride ion-rich solutions (for example, PBS and Saline) 37, 40. In conclusion, HA-pARG NPs are expected to sustain the drug release of cisplatin upon IP administration ${ }^{35}$.

\subsection{Free cisplatin and Cis-pARG-HA NPs exhibit comparable in vitro cytotoxicity}

Upon release of cisplatin from the nanoparticles in the cytosol, cisplatin undergoes an aquation reaction producing highly reactive species $\left[\mathrm{Pt}\left(\mathrm{NH}_{3}\right)_{2} \mathrm{Cl}(\mathrm{H} 2 \mathrm{O})\right]^{+}$or $\left[\mathrm{Pt}\left(\mathrm{NH}_{3}\right)_{2} \mathrm{Cl}(\mathrm{H} 2 \mathrm{O})_{2}\right]^{2+}$, which can enter the nucleus and bind to the $\mathrm{N} 7$ atoms of the purine residue guanine via coordination bonds, giving rise to the formation of intra-strand and inter-strand Pt-DNA cross-links. These DNA adducts interfere in cellular processing of DNA lesions, eventually inducing cell apoptosis ${ }^{41-42}$. To explore the antitumor activity of released cisplatin from Cis-pARG-HA NPs, we first conducted in vitro efficacy studies (Figure 1D). About 99\% of human ovarian cancer cells (SKOV-3) treated with drug-free nanoparticles, at equivalent polymer concentrations to Cis-pARG-HA NPs, remained viable for each of the examined concentrations, pointing to the fact that the polymeric constituents of pARG-HA NPs had no cytotoxic effects on the SKOV-3 cells as such. As shown in Figure 1D, the cytotoxicity was clearly enhanced in a dose-dependent manner for free cisplatin and Cis-pARG-HA NPs. The half-maximal inhibitory concentration $\left(\mathrm{IC}_{50}\right)$ was determined as $28.9 \pm 1.7 \mu \mathrm{M}$ and $30.7 \pm 3.6 \mu \mathrm{M}$ for free cisplatin and CispARG-HA NPs, respectively, demonstrating the anticancer drug efficacy of cisplatin both in its free and encapsulated form. 
A
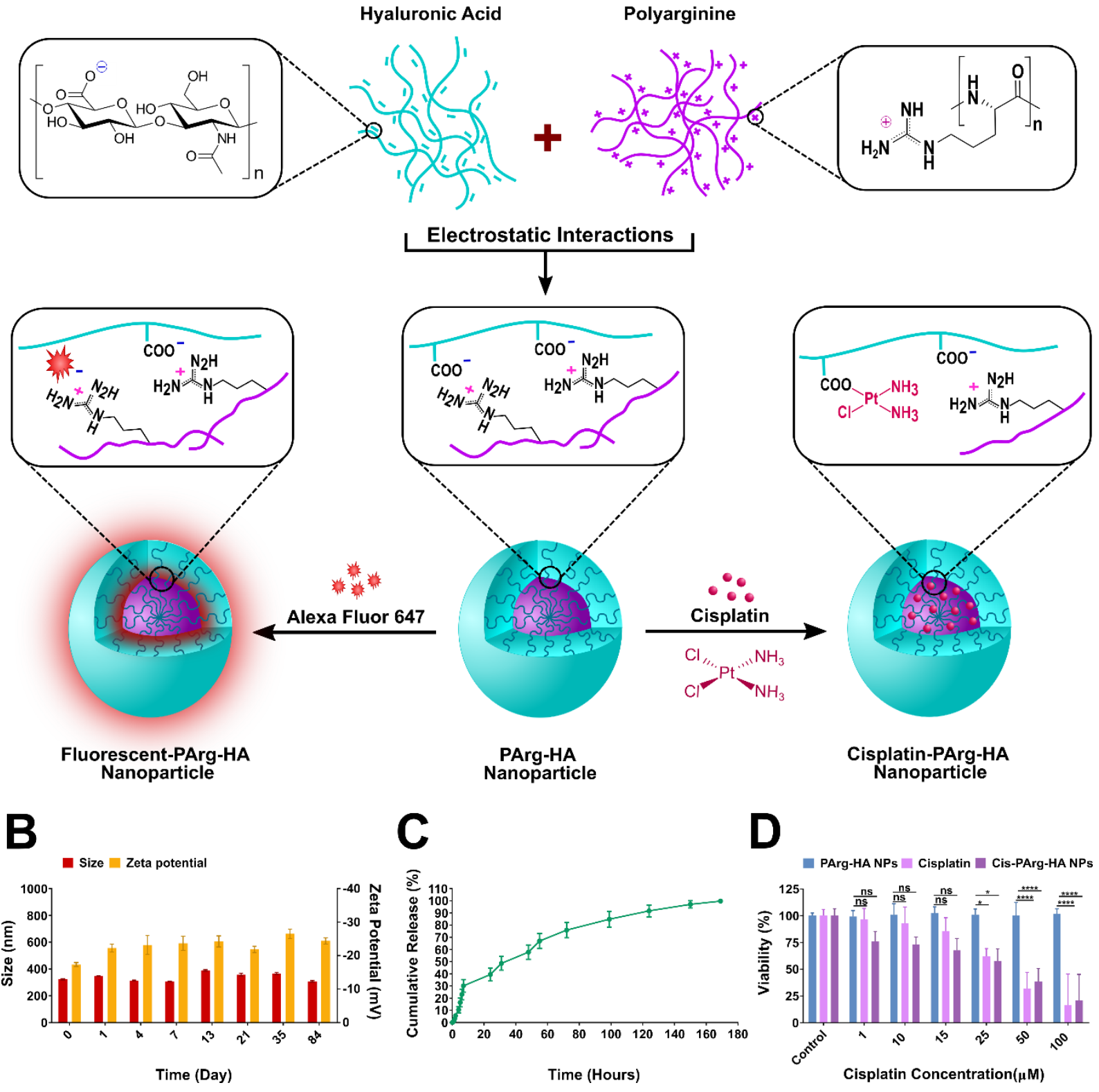

Figure 1: Preparation and characterization of pARG-HA NPs and Cis-pARG-HA NPs. (A) Schematic illustration of the development of pARG-HA NPs based on electrostatic complexation between negatively charged HA and positively charged pARG polymers. pARG-HA NPs can be loaded with cisplatin (Cis-pARG-HA NPs) (right) or fluorescently labeled using Alexa Fluor 647 (F-pARG-HA NPs) (left) by respectively adding cisplatin and Alexa Fluor 647 to the polymer solutions during synthesis. (B) Stability profile of pARG-HA NPs over time in aqueous solution in terms of size and surface charge. (C) Cumulative in vitro drug release kinetics of Cis-pARG-HA NPs over time in phosphate buffered saline (PBS) (pH: 7.4) at $37^{\circ} \mathrm{C}$. (D) Relative viability of human ovarian cancer cells (SKOV-3) following $24 \mathrm{~h}$ incubation with Cis-pARG-HA NPs, pARG-HA NPs and free cisplatin. All experiments were reproduced three times, each time in triplicate. Results are presented as mean \pm SD and statistically significant differences between groups are shown as asterisks: $* \mathrm{P}<0.05$; $* * * * \mathrm{P}<$ 0.0001 ; ns: nonsignificant analyzed by one-way or two-way ANOVA.

\section{4. pARG-HA NPs are colloidally stable in undiluted ascetic fluid}


Colloidal stability is a decisive factor for the success of any nano-sized delivery system upon exposure to physiological conditions within the human body. When a nanoscale system encounters biological environments, various proteins abundant in extracellular fluids can rapidly adsorb onto the NP and cover its surface. This so-called 'protein corona' might highly affect the nanoparticle physicochemical properties (e.g., size, charge and surface features), its stability (e.g., aggregation or premature cargo release) and biological activity (e.g., cellular uptake, intracellular trafficking, biodistribution, toxicity and clearance patterns) ${ }^{43-44}$. Although DLS is the most common technique to determine the size distribution profile of small-sized particles in aqueous media, it should be noted that it typically fails to precisely characterize the NP size in undiluted biological fluids, primarily owing to strong light scattering by the proteins in theses biofluids. We have previously shown, however, that fluorescent Single Particle Tracking (fSPT) is able to examine the colloidal stability of nanoparticle formulations in undiluted biofluids (e.g., ascites, serum, blood and plasma) on a per particle basis ${ }^{45}$. Therefore, we investigated the colloidal stability of the pARG-HA NPs in ascetic fluid, as a relevant physiological in vitro condition for intraperitoneal administration by fluorescent Single Particle Tracking (fSPT) ${ }^{45}$. Fluorescently labeled pARG-HA NP (F-pARG-HA NP) were prepared by the electrostatic encapsulation of Alexa Fluor 647 carboxylic acid, tris (triethylammonium) salt (Figure 1A), resulting in NPs with similar physicochemical characteristics as compared to unlabeled ones (250 $\pm 10 \mathrm{~nm}$ and $23.5 \pm 1.1 \mathrm{mV}$ ). As shown in Figure 2A, the NP size measured with fSPT in distilled water was in line with our DLS results, with a peak diameter around $265 \mathrm{~nm}$. Incubation of F-pARG-HA NPs in $90 \%$ vol. ascetic fluid was accompanied by an increase in size from approximately $250 \mathrm{~nm}$ to $466 \mathrm{~nm}$ at $1 \mathrm{~h}$ postincubation at $37{ }^{\circ} \mathrm{C}$, showing a slight nanoparticle aggregation in ascetic fluid. Of note, it has been recently reported that formation of an ascites-induced protein corona and the subsequent NP size expansion could inhibit undesired drainage through lymphatic duct openings and thus lengthen peritoneal retention ${ }^{46,47}$. Furthermore, only a small fraction of ascites may exist in the peritoneal cavity of patients eligible for CRS and adjuvant IP chemotherapy regimens, considering that all ascetic fluid is drained off the peritoneal space before any IP surgical or chemotherapeutic procedure is pursued. This can in part minimize the protein-nanoparticle interactions in ascites, when nano-sized delivery vehicles are administered to the peritoneal cavity of patients with PC. Taken together, we found a slight aggregation of F-pARG-HA NPs in ascetic fluid, which possibly results from the formation of a protein corona on the NPs surface. In the next section, we investigated whether this protein corona also influences the cellular uptake of the F-pARG-HA NPs.

\section{5. pARG-HA NPs are internalized into SKOV-3 cells through CD44-receptor mediated endocytosis}

In the next set of experiments, we explored whether F-pARG-HA NPs can be efficiently internalized into SKOV-3 cells and whether or not internalization was influenced by 1 hour pre-incubation in $90 \%$ vol. undiluted biofluids such as human serum and ascites. Therefore, F-pARG-HA NPs were incubated in the biological fluids at $37{ }^{\circ} \mathrm{C}$ for 1 hour and NP uptake was subsequently assessed using flow cytometry. Our results confirmed high cellular uptake of F-pARG-HA NPs in Opti-MEM (i.e., lowprotein content), as well as after pre-incubation in human serum and ascites (i.e., high protein concentration), with more than $86 \%$ of the cell population internalizing NPs within the 4 hours of exposure (Figure 2B). The high cell-associated fluorescence intensity for all the examined conditions (i.e., Opti-MEM, serum and ascites) demonstrates that cellular uptake is unaffected in protein-rich conditions. Hence, the protein corona formed around the nanoparticles in biological fluids, if any, did not interfere with the uptake of the NPs by the SKOV-3 cells. Possibly, the negatively charged surface of pARG-HA NPs, protects the nanocarriers not only against aggregation but also limits the interaction between NPs and anionic charged proteins abundant in biological fluids. 
Possibly, HA on the NP surface could act as a targeting ligand that enables the specific uptake by CD44overexpressing tumor cells like SKOV-3 cells ${ }^{24-25,35,48-50}$. To verify this hypothesis, the cellular uptake behavior of F-pARG-HA NPs was studied in SKOV-3 cells in the absence or presence of high concentration of free HA $(10 \mathrm{mg} / \mathrm{mL})$. Pre-exposure of SKOV-3 cells with excess free HA led to a clear decrease in percentage of positive cells as well as fluorescence intensity that may be indicative of blockage of CD44 receptors by free HA (Figure 2C). In the absence of free HA, by contrast, our results revealed that NPs were significantly taken up by the cells, possibly denoting the specific HA-CD44 interactions, facilitating NP cellular internalization (Figure 2C). The successful uptake of F-pARG-HA NPs by SKOV-3 cells through CD44-receptor mediated endocytosis was further confirmed by confocal microscopy, where the high intracellular accumulation of F-pARG-HA NPs was evident from a strong red signal in the cytoplasm (Figure 2D). Conversely, a low level of intracellular fluorescence was monitored (a weak red signal) in the cytosol of free HA-treated SKOV-3 cells, again confirming that saturation of the CD44 receptors with free HA to a great extent diminished the cellular internalization. Therefore, both flow cytometry and confocal microscopy confirm that F-pARG-HA most likely entered the SKOV-3 cells in a target-specific manner via strong binding affinity of HA to the CD44 receptor. To further confirm that F-pARG-HA NPs uptake proceeds through the CD44 receptor, competitive inhibition study was performed with an additional cell line. Therefore, the 3T3 (mouse fibroblast) cell line was used, that does not express high levels of CD44 receptors, when compared to SKOV-3 cells. Our results demonstrate low mean fluorescence intensity in 3T3 cells, indicating low cellular uptake of F-NPs by 3T3 cells both in the presence and absence of free excess HA. In SKOV-3 cells, however, a high mean fluorescence intensity (reflecting high cellular uptake) is observed, that decreases in the presence of high concentration of free HA. This in turn denotes the CD44 mediated uptake of pARGHA NPs by SKOV-3 cells (Figure S1). 
A
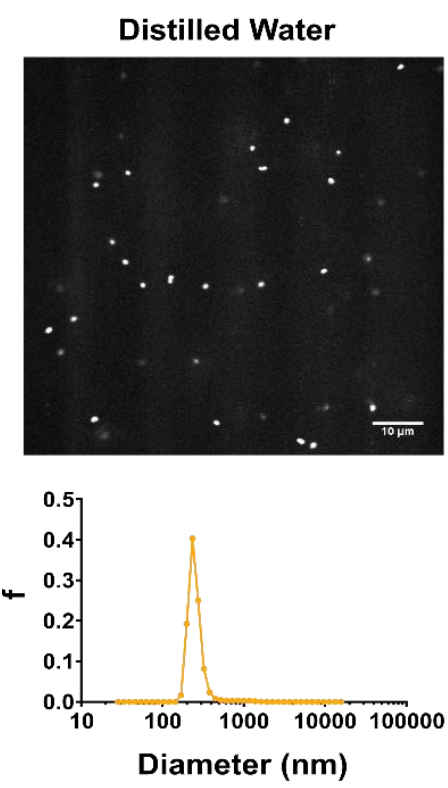

Average d: $265.7 \mathrm{~nm}$
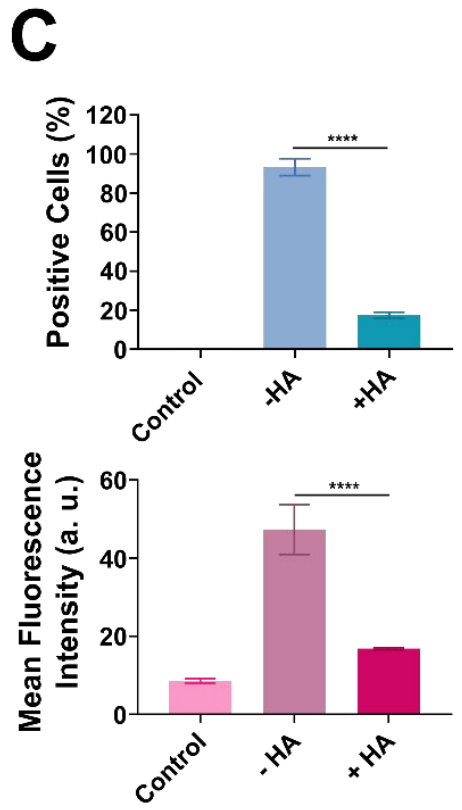

Ascites
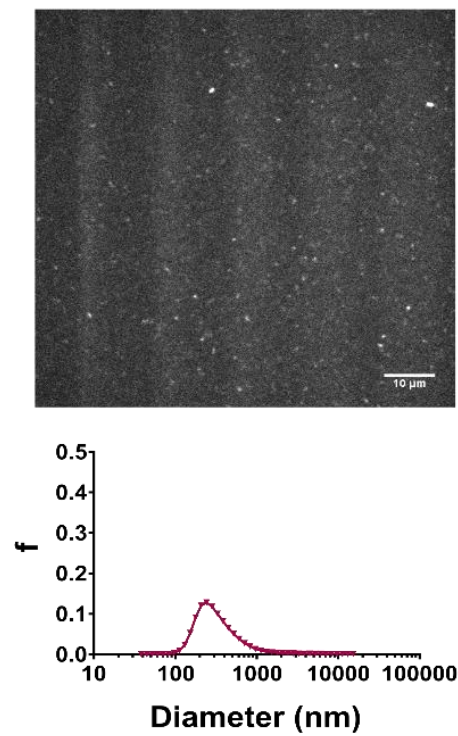

Average d: $466 \mathrm{~nm}$
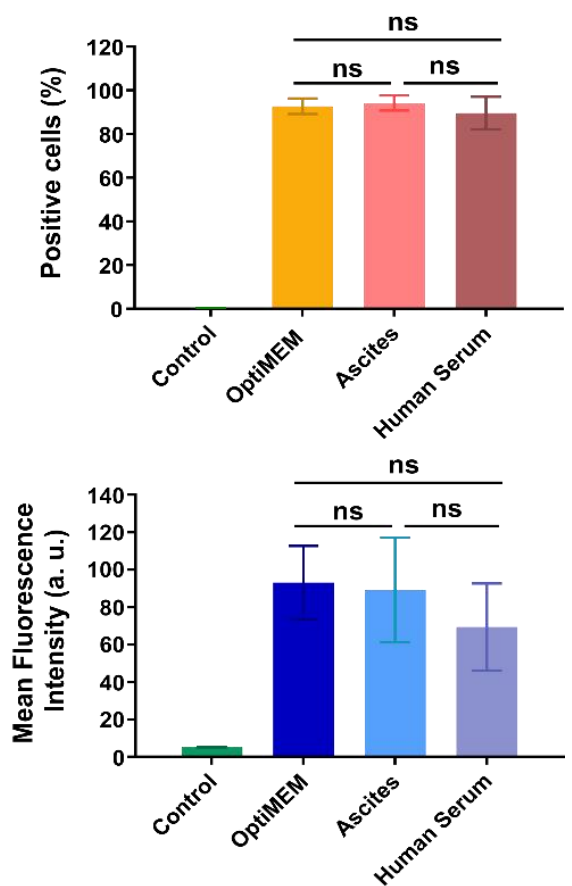

D

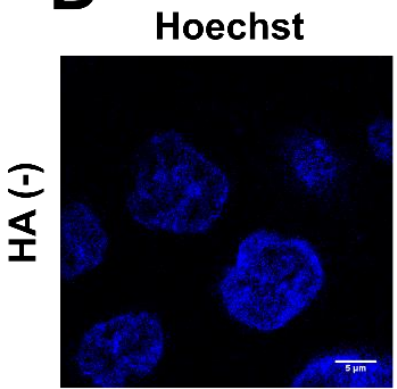

\section{Alexa Fluor 647}
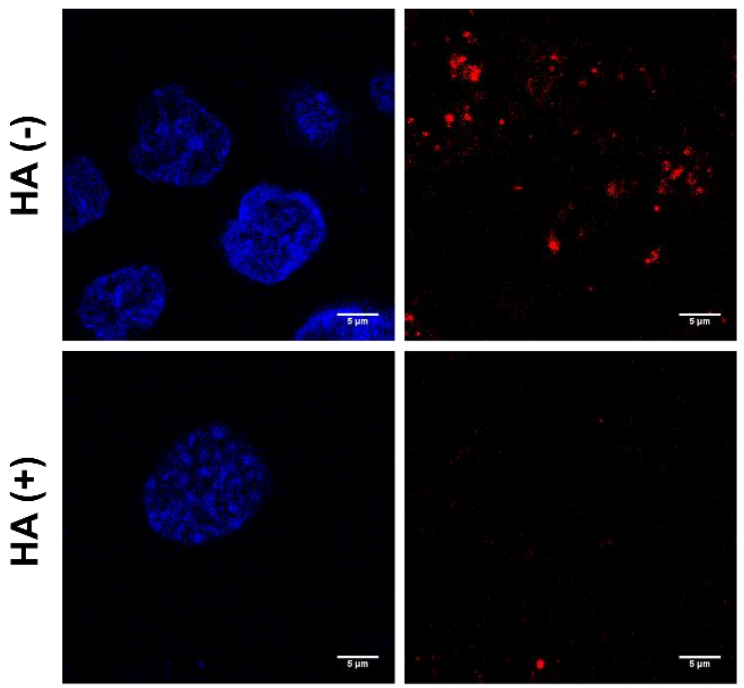
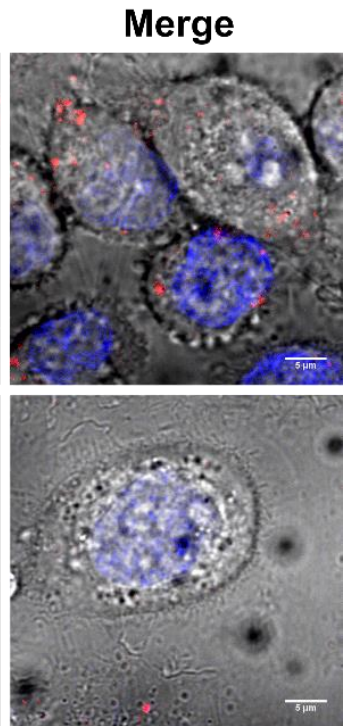

Figure 2. (A) In vitro colloidal stability of pARG-HA NPs in the presence of biofluids. Snapshots and size distribution profile of pARG-HA NPs following $1 \mathrm{~h}$ incubation in distilled water and $90 \%$ vol. undiluted human ascites at $37{ }^{\circ} \mathrm{C}$, obtained by fSPT analysis. In the fSPT size distributions, Y-axis points to the frequency (f) of nano-sized particles observed with the corresponding size on the $\mathrm{X}$-axis. (B) In vitro uptake of pARG-HA NPs by SKOV-3 cells in Opti-MEM (i.e., low-protein content), as well as $90 \%$ vol. undiluted human serum and ascites (i.e., protein-rich condition) $4 \mathrm{~h}$ after exposure. The cellular uptake behavior of pARG-HA NPs was quantified based on percentage of positive cells and mean fluorescence signal intensity in all examined conditions. (C) In vitro cellular internalization of pARG-HA NPs through CD44-receptor mediated endocytosis in the presence or absence of excess free 
HA, assessed by flow cytometry analysis and (D) confocal microscopy (blue: nucleus, red: F-pARGHA NPs). All experiments were reproduced three times, each time in triplicate. Results are presented as mean \pm SD and statistically significant differences between groups are shown as asterisks: $* * * * \mathrm{P}<$ 0.0001; ns: nonsignificant analyzed by one-way or two-way ANOVA.

\subsection{Aerosolization of Cis-pARG-HA NPs synergistically results in a pronounced antitumor} efficacy in a rat model of peritoneal metastasis

Locoregional intraperitoneal chemotherapy has the potential to concentrate anticancer therapeutics at the tumor site, resulting in enhanced local antitumor responses and minimized systemic adverse effects ${ }^{6,51}$. In the pursuit of developing new IP drug delivery strategies for PC therapy, a minimally invasive, intra-abdominal aerosol-based therapy, referred to as PIPAC, has been recently introduced ${ }^{11}$. By harnessing the physical properties of gas and pressure, PIPAC delivers cytotoxic agents in the form of a pressurized aerosol to the peritoneal space, thereby warranting a more homogeneous drug distribution and a deeper drug penetration into peritoneal tumor nodules within the abdominal cavity ${ }^{52-53}$. Also nanotechnology-based drug delivery systems present several prominent features for locoregional drug delivery, such as extended peritoneal retention and targeted drug delivery towards the desired tumor tissue ${ }^{15-16}$. Thus far, however, only a very few studies have been devoted to examine the in vivo feasibility and therapeutic benefits of IP aerosolization of nanoparticle-based cancer therapeutics ${ }^{54} 55$. Therefore, the feasibility and added benefits of the PIPAC therapy with nanoparticles still largely remain to be elucidated.

In this study, we explored the in vivo potency of cisplatin-loaded pARG-HA NPs in a rat model of intraperitoneally disseminated human ovarian cancer ${ }^{56-57}$, with a special focus on the potential advantage of formulating cisplatin into a nanoparticle system and whether or not liquid aerosolization is beneficial compared to liquid injection of NPs into the peritoneal cavity. Tumor-bearing rats were dosed either with free cisplatin $(5 \mathrm{mg} / \mathrm{kg}$ ) or cisplatin-loaded pARG-HA NPs (at $5 \mathrm{mg} / \mathrm{kg}$ doseequivalent level of $\mathrm{Pt}$ ) through a single injection or nebulization (PIPAC) (Figure 3A). In the control group, rats were IP injected with saline $(0.9 \% \mathrm{NaCl})$ alone. The rat is a well-suited animal model for the PIPAC procedure ${ }^{54,57}$, as the adequate space of the peritoneal cavity allows PIPAC to be effectively performed in clinically relevant conditions. Figure $3 \mathrm{~B}$ illustrates the surgical procedure of the PIPAC method in a rat model of peritoneal surface malignancies, which was conducted during laparoscopy. The nebulization was monitored with a camera, to confirm the formation of an aerosol within the closed space of the peritoneal cavity.

Upon IP delivery of free cisplatin or Cis-pARG-HA NPs, tumor growth and progression of metastasis were continuously monitored utilizing serial bioluminescence imaging for 3 weeks post-treatment (Figure 3C and Figure S2). Furthermore, the average tumor weight (Figure 3E) and spread of metastasis in the peritoneal cavity (Figure 3F) was determined, together with the overall survival of animals in the different treatment groups (Figure 3G). Control animals (treated with saline), showed an aggressive tumor growth, leading to a rapid spread of tumor nodules over the entire peritoneal membrane within 8 days, along with a dramatic increase in the corresponding average radiance value of tumors (Figure 3D, green line). Consequently, all animals had a high tumor weight (Figure 3E, green dots) and reached the humane endpoint at maximum 8 days following treatment (Figure 3G, green line). Animals that were treated with free cisplatin, either through IP injection (dark blue line) or nebulization (light blue line), exhibited a minimal reduction in their tumor burdens 4 to 8 days post-administration, as compared to those receiving saline alone. Although there seems to be a small delay in tumor growth, as evidenced by Figure 3D and 3E, animals again reached the humane endpoint by maximally day 15 post treatment (Figure 3G, dark and light blue lines). Therefore, it appears that a single IP administration of cisplatin is only able to reduce the tumor growth rate for a short period of time, and peritoneal tumors are prone 
to rapid relapse immediately after cisplatin is cleared from the peritoneal cavity and absorbed into the systemic circulation (due to its small molecular size). Interestingly, for the group receiving IP injected Cis-pARG-HA NPs, tumor progression was remarkably delayed for the first 8 days following treatment, in contrast to free cisplatin (Figure 3D, purple line compared to dark and light blue line) and saline groups (Figure 3D, green line). Although the tumor growth resumed by the $14^{\text {th }}$ day post-injection, the maximal average radiance of tumors was much lower than those of rats injected with free cisplatin or saline. Therefore, it seems that encapsulating the cisplatin into pARG-HA NPs can lengthen the residence time in the peritoneal cavity and simultaneously provide sustained drug release to the tumor site, corresponding to the sustained release of cisplatin from pARG-HA NPs over 8 days as found in the in vitro release study (Figure 1C). More intriguingly, the good anti-tumor efficacy even further improved when Cis-pARG-HA NPs were nebulized in the peritoneal cavity of the tumor bearing rats. Indeed, a nearly complete inhibition of tumor regrowth was observed within 21 days post-treatment, demonstrating a synergistic anticancer effect of the combinatorial application of cisplatin-loaded nanoparticles and the PIPAC technique (Figure 3D, orange line). The superior anti-tumor activity of cispARG-HA NPs both after injection and (even more) nebulization is also evident from the weight of intra-abdominal nodules. As shown in Figure 3E, the average tumor weight of rats treated with saline, IP injected cisplatin and IP nebulized cisplatin was found to be $5.5 \pm 0.9 \mathrm{~g}, 4.2 \pm 0.9 \mathrm{~g}$ and $3.4 \pm 0.5 \mathrm{~g}$ per rat, respectively, resulting in no statistically significant difference between these 3 treatment groups. Both injected and nebulized Cis-pARG-HA NPs, however, exhibited a notable decrease in the average weight of total tumor nodules isolated from the peritoneal cavity, with $1.2 \pm 1.1 \mathrm{~g}$ and $0.07 \pm 0.06 \mathrm{~g}$ per rat for IP injection and nebulization, respectively (Figure 3E). Moreover, the extent of tumor dissemination in the peritoneal cavity of animals sacrificed at their humane endpoint confirmed the superior activity of nebulized Cis-pARG-HA NPs (Figure 3F). In the saline and IP injected/nebulized cisplatin groups, numerous metastatic nodules were found all over the intestine and mesentery (Figure 3F, closed black lines). IP injection of Cis-pARG-HA NPs, retarded the spread of tumor nodules on the peritoneum, as only a few small disseminated nodules remained, whereas nebulization of Cis-pARGHA NPs even led to no or nearly undetectable levels of tumor nodules in the peritoneal cavity (Figure 3F). Collectively, our results indicate that Cis-pARG-HA NPs are highly effective in delivering cisplatin to tumors in the peritoneal cavity and can withstand the forces and high pressure generated during the aerosolization procedure (as confirmed by their in vitro colloidal stability upon nebulization (Figure S3)), ultimately leading to a $100 \%$ survival rate during the 21 -day observation period. 

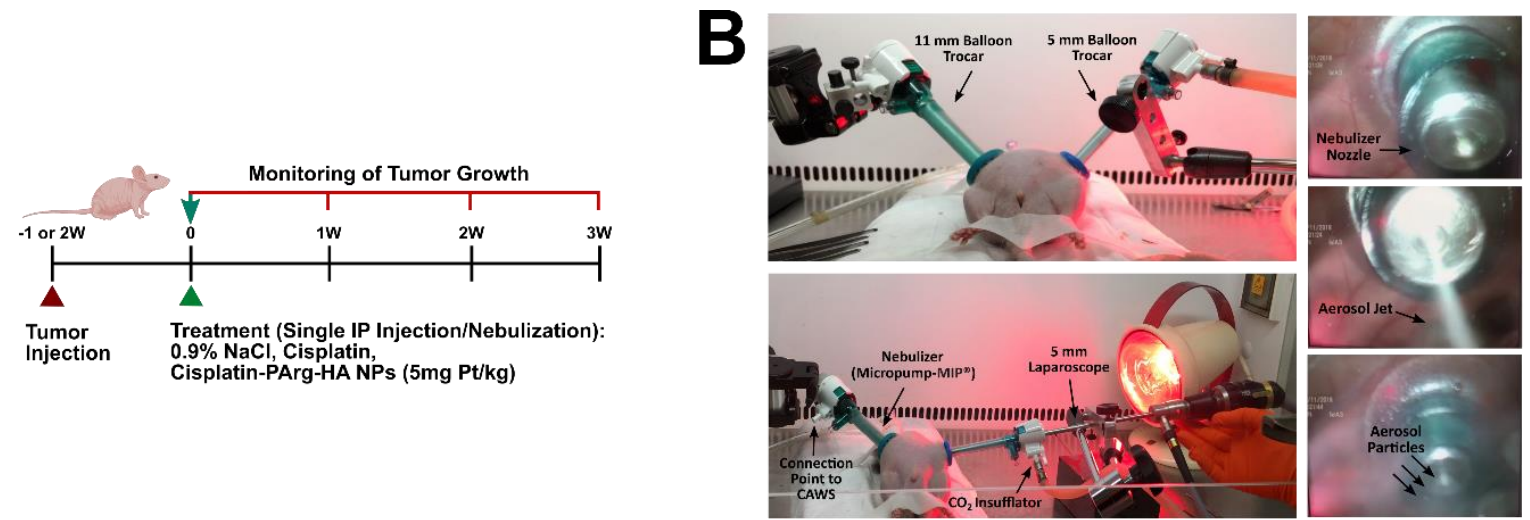

C
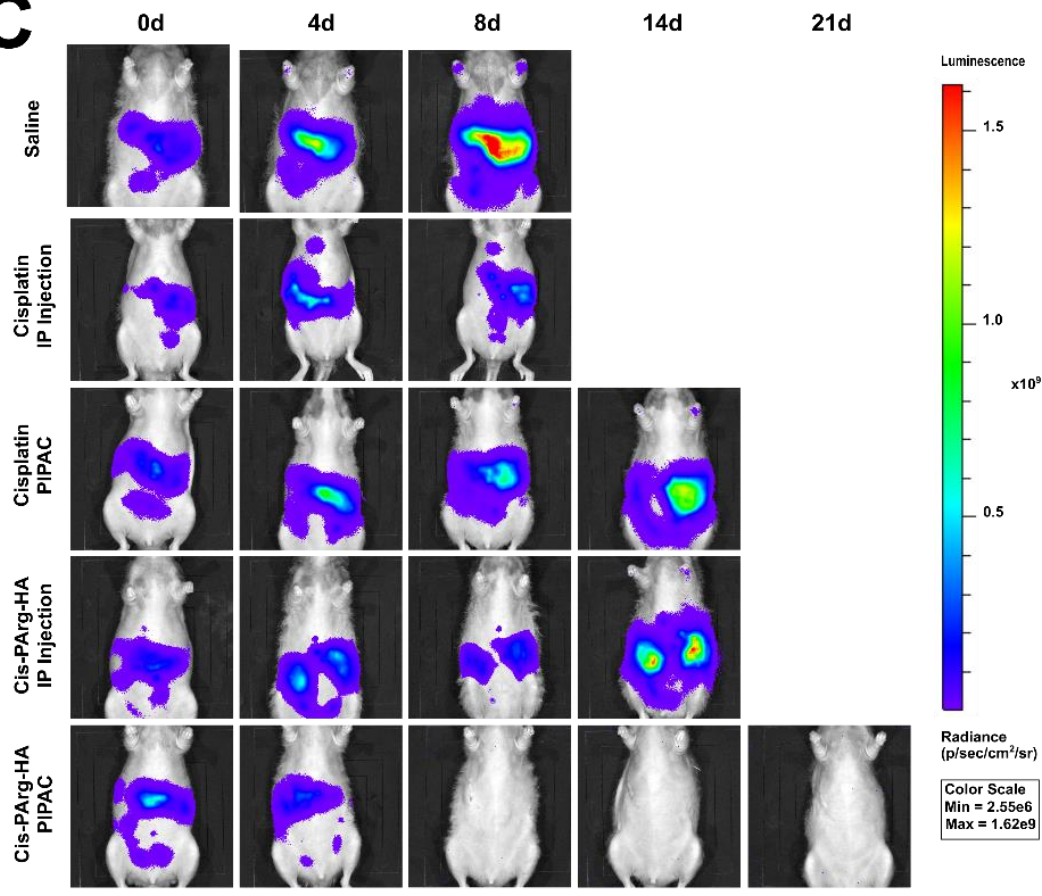

F

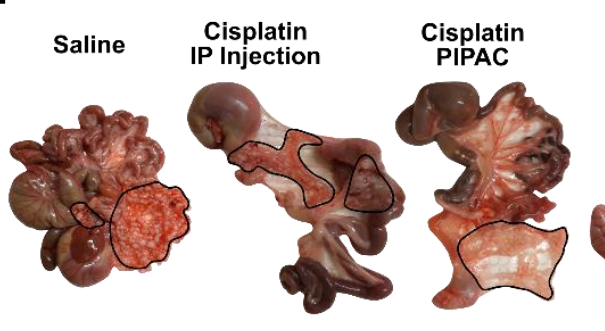

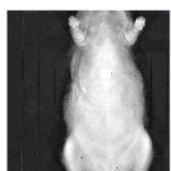

14d

Cis-PArg-HA IP Injection

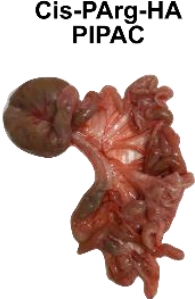

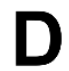
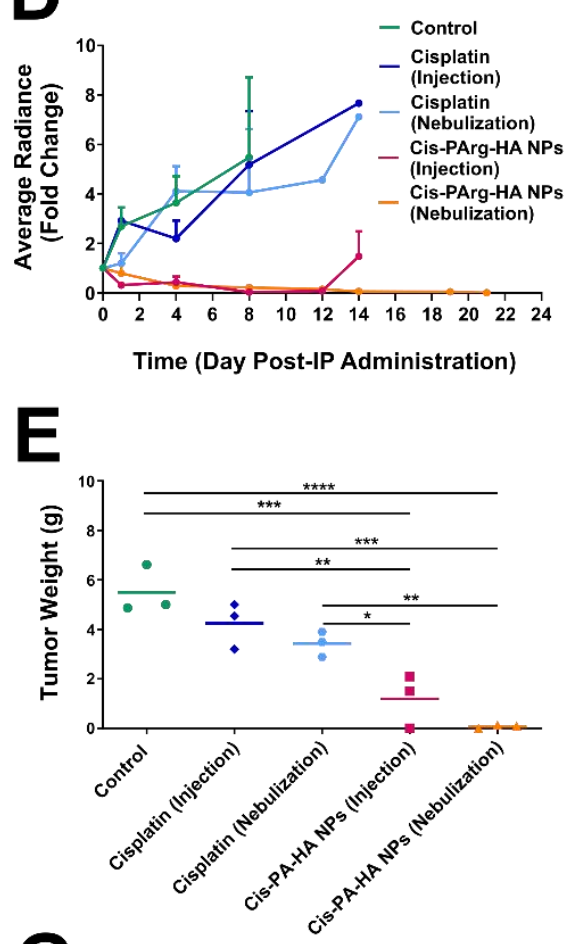

G

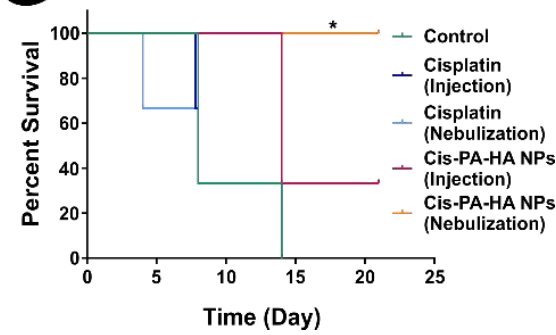

Figure 3. In vivo antitumor efficacy of cisplatin-loaded pARG-HA NPs in a rat model of intaperitoneally disseminated human ovarian cancer upon IP injection and IP nebulization (PIPAC). (A) Treatment schedule adopted in this study. (B) Schematic illustration of surgical procedure of the PIPAC method in a tumor bearing rat, performed during a short laparoscopic procedure. Video monitoring is provided by a $5 \mathrm{~mm}$ camera (laparoscope), confirming the formation of aerosol particles within the closed space of the peritoneal cavity. CAWS, closed aerosol waste system. (C) The antitumor activity of different IP therapeutic strategies according to whole-body bioluminescence images of animals in each treatment group. The most representative animal in each group is presented (the complete overview of animals 
can be found in Figure S2). Tumor growth and progression of metastasis were continuously monitored utilizing serial bioluminescence imaging for 3 weeks post-treatment. (D) Tumor growth profile of each treatment group over time, as determined according to changes from baseline average radiant flux associated with the bioluminescence signal intensity. (E) Weight of intra-abdominal tumor nodules per rat. (F) Representative photographs of extent of tumor dissemination in the peritoneal cavity of animals that reached the humane endpoint (on average, 8, 14 and 21 days post-IP administration for saline/free cisplatin, injected Cis-pARG-HA NPs and nebulized Cis-pARG-HA NPs respectively) . The closed black lines indicate the area that metastatic peritoneal nodules are located. $(\mathrm{G})$ Overall survival curves of the tumor bearing rats in each treatment group. All experiments were reproduced three times. Results are presented as mean $\pm \mathrm{SD}(\mathrm{n}=3-4)$ and statistically significant differences between groups are shown as asterisks: $* \mathrm{P}<0.05 ; * * \mathrm{P}<0.01 ; * * * \mathrm{P}<0.001 ; * * * * \mathrm{P}<0.0001$ analyzed by one-way or two-way ANOVA.

\subsection{PIPAC may improve the in vivo accumulation and penetration of pARG-HA NPs into peritoneal tumor nodules}

To gain a better insight into the mechanism underlying the in vivo potency of the Cis-pARG-HA NPs, fluorescently labeled pARG-HA NPs were intraperitoneally injected or nebulized in tumor baring rats and biodistribution and tumor penetration was assessed 48 hours after IP administration. The homogeneity of the distribution of NPs was evaluated by harvesting tumor nodules from different regions of the peritoneal cavity as illustrated in Figure 4A. IP aerosolized pARG-HA NPs more effectively accumulated at the edge of the peritoneal nodules located in the regions directly opposite and around the MIP ${ }^{\circledR}$ nozzle (nodules $\mathrm{c}$ and d, with fluorescence intensities between 200 and 250 a.u.), while nodules a and $\mathrm{b}$, located more distant from the aerosol jet displayed a lower overall fluorescence intensity (between 100 and 150 a.u.) (Figure 4A). With regard to NPs delivered via IP injection, both tumor nodules harvested from random locations in the peritoneal space (namely region e and f), showed identical results regarding tumor accumulation and fluorescence signal intensity of pARG-HA NPs (with average fluorescence intensities between 100 and 150 a.u). Our findings are consistent with previous studies indicating that the intra-abdominal drug distribution after PIPAC is not as homogeneous as expected but varies between different regions of the peritoneal cavity ${ }^{58}$. As validated by granulometric analysis, about 97.5 vol. $\%$ of the aerosol droplets delivered by the PIPAC micropump are not submicronic (size range between 3-200 $\mu \mathrm{m}$ ) and consequently do not have the ideal physical properties to distribute homogenously. As a result, such aerosol droplets are primarily deposited in the areas on the opposite side of the MIP ${ }^{\circledR}$ nozzle outlet because of gravitational settling and inertial impaction, thereby forming local "hotspots" on the peritoneum beneath the MIP ${ }^{\circledR}$ and in areas lateral to the aerosol jet ${ }^{59-62}$. The same holds true for in-tissue drug penetration depth provided by PIPAC, where a deeper penetration of aerosolized drugs into the tissue $(>311 \mu \mathrm{m}$ in depth) was observed in the local hotspots created on the peritoneum in the vicinity of the $\mathrm{MIP}^{\circledR}{ }^{61-63}$. Also, we observed that the majority of IP-nebulized NPs were largely localized at the edge of the tumor tissue opposite the nebulizer with a maximum penetration depth of approximately $200-300 \mu \mathrm{m}$. This penetration depth might be sufficient, however, to prevent cancer recurrence, as most tumor nodules that remain in the peritoneal cavity after cytoreductive surgery are microscopic tumors nodules.

Quantitative analysis of the fluorescence signal intensity emanating from F-pARG-HA NPs in the tumor tissue sections revealed that, on average, identical fluorescent signals were obtained in the IP cavity of rats receiving pARG-HA NPs through injection or nebulization (Figure 4B, light green bars). The maximum fluorescence intensity that was observed in tissue sections, however, was always higher for IP nebulization, in comparison to IP injection (Figure 4B, dark green bars). This confirms that IP aerosolized delivery of pARG-HA NPs overall deposits higher nanoparticle concentrations on the tumor 
nodules, when compared to IP injection, resulting in the formation of more highly intense NP hotspots. Of note, a low background signal was detected in the control tissues such as liver, spleen and kidney (Figure 4C), indicating that pARG-HA NPs preferentially homed to tumor nodules and were not readily cleared from the peritoneal cavity and subsequently trafficked into filtering organs (e.g., spleen and liver). This biodistribution was also confirmed using a rat model with one big tumor nodule, instead of numerous small peritoneal tumor nodules (Figure S4). As supported by our data on in vitro cellular uptake, it is likely that the preferential accumulation of pARG-HA NPs in the tumor tissue stems from the CD44-receptor targeting ability of NPs conferred by HA exposed on the NP surface as a targeting ligand. Collectively, intraperitoneally administered pARG-HA NPs revealed tumor targeting ability, preferential tumor accumulation and penetration into the metastatic tumor nodules.

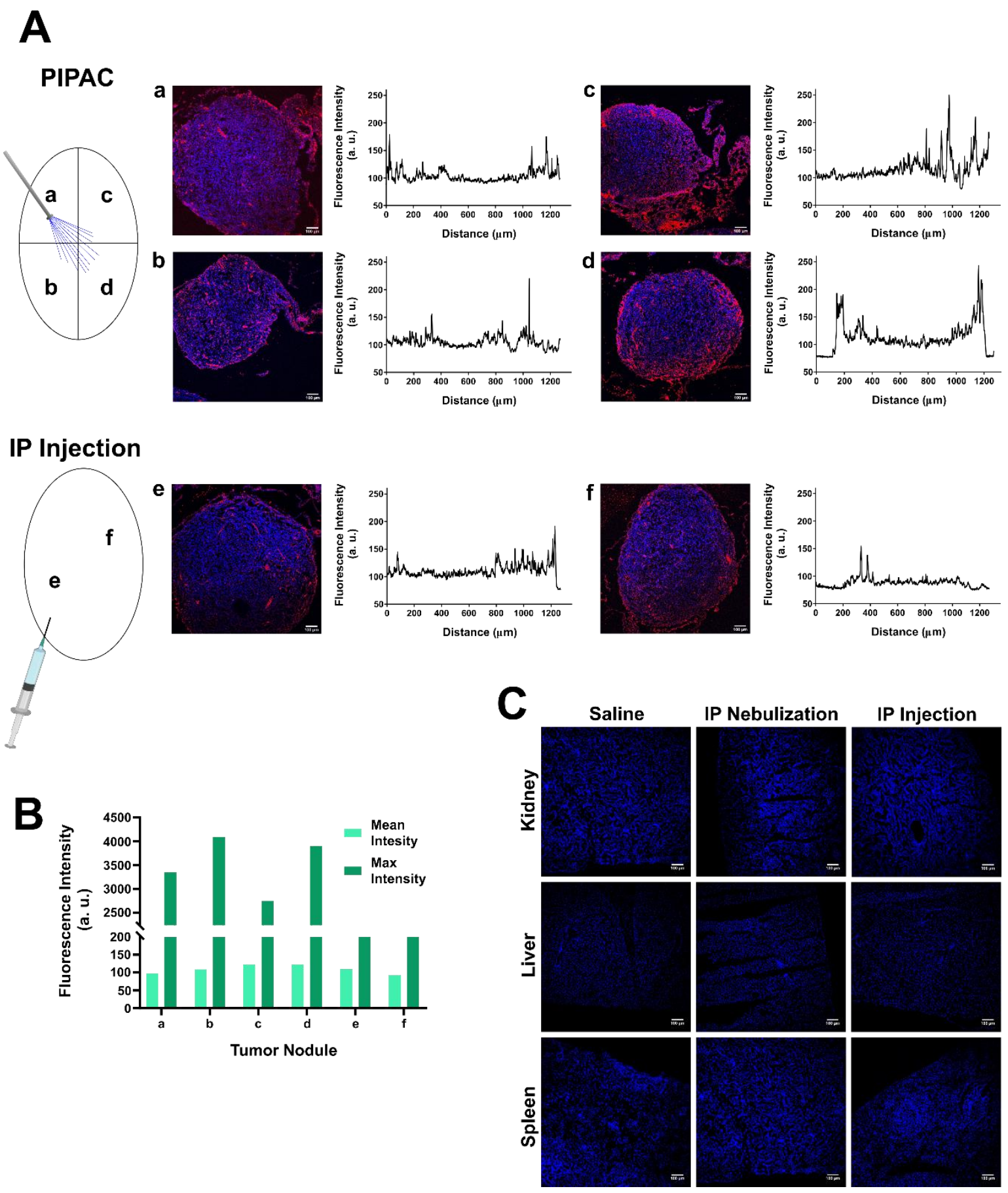


Figure 4. Tissue biodistribution and tumor penetration of intraperitoneally nebulized and injected fluorescently labeled pARG-HA NPs in the female nude rats bearing IP disseminated tumors, 48 hours after IP administration. (A) Representative fluorescence confocal images and corresponding fluorescent intensity distributions of tumor tissue sections excised from different locations in the peritoneal cavity with respect to proximity to the nebulizer (Micropump-MIP ${ }^{\circledR}$ ) outlet (regions $\mathrm{c}$ and d), or distant from the created aerosol jet (regions a and b) in the PIPAC method, as well as random locations in the peritoneal space (regions e and f) for IP injection. (B) Quantification of the fluorescence signal intensity of F-pARG-HA NPs in tissue sections of peritoneal nodules following IP nebulization and injection. The fluorescence signal intensity is expressed as an average and maximum signal intensity per tumor nodule. (C) representative confocal images of tissue sections of control organs (i.e., kidney, liver and spleen) harvested upon IP nebulization and injection of F-pARG-HA NPs along with IP injection of saline as a control. All experiments were reproduced three times $(n=3-4)$.

\section{Conclusions}

Our study offers a starting point for the combinatorial application of cancer nanotherapies and the PIPAC procedure by presenting the therapeutic benefits of IP aerosolization of cisplatin-loaded pARGHA NPs in a rat model of IP disseminated human ovarian cancer. pARG-HA NPs were efficiently loaded with cisplatin, demonstrated colloidal stability in ascetic fluid and internalized into SKOV-3 cells through CD44 targeting mediated by the HA surface of the nanoparticles. Furthermore, cisplatin-loaded nanoparticles showed an enhanced antitumor activity in a rat model of peritoneal carcinomatosis when compared to the injection or nebulization of free cisplatin. The therapeutic potential was even further enhanced by the nebulization of pARG-HA NPs into the peritoneal cavity, demonstrating the synergistic effect of nebulization and encapsulation of cisplatin into a nanoparticle system. The sustained release of cisplatin from the pARG-HA NPs, together with the preferential accumulation of pARG-HA NPs in the metastatic tumor nodules opposite the nebulizer nozzle, most likely contribute to the observed synergistic effect. Overall, our results suggest the potential advantages of IP aerosolization of pARGHA NPs over injection, although there is still significant room for improvement of the delivery of cancer nanotherapeutics via the PIPAC technique to achieve a more homogeneous tissue distribution and enhanced penetration depth. For example, the recent development of new PIPAC methods such as hyperthermic intracavitary nanoaerosol therapy (HINAT) ${ }^{64}$ and electrostatic PIPAC (ePIPAC) ${ }^{65}$ might yield a more uniform intra-abdominal drug distribution pattern and a deeper penetration of drug into the tissue, compared to the conventional PIPAC method. In addition, future studies are needed to understand whether or not repetition of the PIPAC therapy itself or in combination with other controlled release delivery platforms (for example, in-situ cross-linkable hydrogels) is feasible to maintain the therapeutic concentration of Cis-pARG-HA NPs within the peritoneal space over prolonged periods of time.

\section{Materials and Methods}

\subsection{Materials}

Cisplatin (cis-diamminedichloroplatinum (II)) was purchased from Abcam (Cambridge, UK). Poly-LArginine (PA) hydrochloride ( $\mathrm{Mw}=5800 \mathrm{Da})$ was purchased from Alamanda ${ }^{\circledR}$ Polymers (Huntsville, Alabama, USA). Hyaluronic acid (HA) $(\mathrm{Mw}=20 \mathrm{kDa})$ was purchased from Lifecore ${ }^{\circledR}$ Biomedical (Chaska, Minnesota, USA). McCoy's 5A modified medium, Fetal Bovine Serum (FBS), penicillinstreptomycin $(5000 \mathrm{U} / \mathrm{ml}), 0.25 \%$ Trypsin-EDTA (1×), Dulbeco Phosphate buffered Saline (DPBS), Opti-MEM, Alexa Fluor 647 carboxylic acid, TRIS (triethylammonium) salt and Hoechst 33342 were purchased from Invitrogen (Merelbeke, Belgium). 3-(4,5-Dimethyl-2-thiazolyl)-2,5-diphenyl-2H- 
tetrazolium bromide (MTT) and Paraformaldehyde (PFA) were purchased Sigma Aldrich ${ }^{\circledR}$ (St. Louis, Missouri, USA). 0.9\% Sodium Chloride ( $\mathrm{NaCl}$ ) was purchased from B. Braun (Melsungen, Germany).

\subsection{Animals}

All animal studies were conducted under approved protocols by the Animal Ethics Committee of the Faculty of Medicine and Health Sciences, Ghent University, Belgium (ECD 18/23), and in compliance with the Belgian Council for Laboratory Animal Science (BCLAS) guidelines for the Care and Use of Laboratory Animals. Athymic female nude rats (Foxn1: nu/+) (8-10 weeks, $\geq 200 \mathrm{~g}$ ) were purchased from Envigo (Horst, the Netherlands) and housed in the animal core facility. All animals were maintained under controlled conditions with a $12 \mathrm{~h} \mathrm{~h}$ light/dark schedule and food and water ad libitum and acclimatized to standard housing conditions for at least one week prior to experiments.

\subsection{Development and characterizations of blank, fluorescently labeled and cisplatin-loaded Par- HA NPs}

pARG-HA nanosystems composed of hyaluronic acid (HA) and polyarginine hydroxide (pARG-OH) were synthetized via the ionic gelation method (HA/pARG mass ratio: 4.5) ${ }^{27-28}$. pARG-OH was obtained from polyarginine chloride (pARG-Cl) using an Amberlite ${ }^{\circledR}$ IRA $900 \mathrm{Cl}$ ion exchange resin (Sigma Aldrich $^{\circledR}$, St. Louis, MO, USA). To prepare the pARG-OH, NaOH (1M, $3 \mathrm{~mL}$ ) was added to an ion exchange column containing $1 \mathrm{~mL}$ of wet resin. After 30 minutes, the column was rinsed with MilliQ water to adjust the $\mathrm{pH}$ to 7 and the pARG-Cl solution $(50 \mathrm{mg} / \mathrm{mL}, 1 \mathrm{~mL})$ was subsequently added to the column, followed by rinsing the column with $3 \mathrm{~mL}$ of MilliQ water. Finally, the pARG-OH at the concentration of $12.5 \mathrm{mg} / \mathrm{mL}$ (pH: 10-12) was obtained and stock solution was stored at $4{ }^{\circ} \mathrm{C}$ afterwards. Prior to preparation of nano-sized systems, all solutions were filtered through a $0.22 \mu \mathrm{m}$ filter. To synthetize the blank pARG-HA NPs, briefly, $80 \mu \mathrm{l}$ of pARG-OH solution $(2.5 \mathrm{mg} / \mathrm{ml})$ and $120 \mu \mathrm{l}$ of distilled water were mixed in a glass amber vial by magnetic stirrer. Then, $100 \mu \mathrm{l}$ of HA solution $(9 \mathrm{mg} / \mathrm{ml})$ was added to the middle of vortex and the dispersion was kept under magnetic stirring for 10 minutes. Cis-pARG-HA and F-pARG-HA NPs were developed with the same method as described for blank NPs, but instead of distilled water, $120 \mu \mathrm{L}$ of cisplatin solution $(1 \mathrm{mg} / \mathrm{mL})$ or Alexa Fluor 647 carboxylic acid, tris (triethylammonium) salt $(0.8 \mathrm{mg} / \mathrm{ml})$ (Invitrogen, Merelbeke, Belgium) were added to the pARG-OH solution for development of cisplatin-loaded and fluorescently labeled nanoparticles, respectively. Upon completion of NP synthesis, all prepared mixtures were subjected to centrifugal separation to ensure the removal of non-complexed constituents such as free cisplatin, Alexa fluor 647, pARG-OH and HA polymers. To isolate the developed nanoparticle formulations, $300 \mu \mathrm{l}$ of the nanocarrier dispersion was transferred to an Eppendorf microtube containing $20 \mu \mathrm{l}$ of glycerol and centrifuged at $16000 \mathrm{~g}$ for 30 minutes at $25^{\circ} \mathrm{C}$. Next, the supernatant was discarded and the pellet was resuspended in distilled water through vigorous vortexing. The nanoformulations were stored at $4^{\circ} \mathrm{C}$ afterwards. All nanoformulations were further diluted in distilled water to a final volume of $1000 \mu \mathrm{L}$ and size and zeta potential were subsequently measured by Zetasizer Nano-ZS (Malvern, Worcestershire, UK). Following encapsulation of cisplatin in pARG-HA NPs drug loading content and encapsulation efficiency were analyzed by ICP-MS and calculated according to the equations as follows:

Drug Content $(\%)=\frac{\text { Weight of cisplatin in nanoparticles }}{\text { weight of polymers fed initially }} \times 100$

Encapsulation Efficiency $(\%)=\frac{\text { Weight of cisplatin in nanoparticles }}{\text { weight of cisplatin fed initially }} \times 100$

\subsection{In vitro release kinetics}

$1 \mathrm{~mL}$ of Cis-pARG-HA NPs dispersion $(0.36 \mathrm{mg} \mathrm{Pt})$ was placed in a Float-A-Lyzer ${ }^{\circledR} \mathrm{G} 2$ dialysis tube (MWCO: $3.5-5 \mathrm{kDa}$ ) (Spectrum Laboratories, California, USA) and immersed in $75 \mathrm{~mL}$ of PBS (pH: 
7.4), stirring constantly at $37{ }^{\circ} \mathrm{C}$ for 1 week. At given time points, samples $(1 \mathrm{~mL})$ were collected from the release medium and replaced with fresh PBS. Pt content was determined using ICP-MS afterwards.

\subsection{Collection of biological fluids}

To obtain the human serum, blood was withdrawn from healthy volunteers and collected into VenosafeTM tubes $(6 \mathrm{~mL})$ containing gel and clotting activator (Terumo EuropeTM, Leuven, Belgium) at Ghent University Hospital. Thereafter, the tubes were centrifuged $\left(4000 \mathrm{~g}, 10 \mathrm{~min}, 20^{\circ} \mathrm{C}\right)$ and serum (supernatant) was transferred into microvette ${ }^{\circledR} 500$ Z-Gel (SARSTEDT, Numbrecht, Germany) and subjected to centrifugation $\left(10000 \mathrm{~g}, 5 \mathrm{~min}, 20^{\circ} \mathrm{C}\right)$. The obtained serum was then stored at $-20{ }^{\circ} \mathrm{C}$. Furthermore, human ascites was obtained from patients with peritoneal carcinomatosis at the department of medical oncology, Ghent University Hospital, according to an approved procedure by the ethics committee of the Ghent University Hospital (EC no. 2013/589).

\subsection{In vitro colloidal stability of pARG-HA NPs in biological fluids}

Fluorescent Single Particle Tracking, a well-suited advanced fluorescence microscopy technique, enables us to precisely examine the colloidal stability of nano-sized delivery systems in undiluted biological fluids (e.g., ascites, serum, blood and plasma) on a per particle basis, as shown previously ${ }^{66}$. In short, fSPT exploits an iXon ultra EMCCD camera (Andor Technology, Belfast, UK) and a sweptfield confocal (SFC) microscope (Nikon eclipse Ti, Japan) equipped with an MLC 400 B laser (Agilent technologies, California, USA) to record high-speed movies of individual fluorescently labeled particles diffusing in biofluids. Captured videos are then analyzed by in-house image processing software, obtaining a distribution of diffusion coefficients which is then converted into a size distribution utilizing the Stokes-Einstein equation, considering the viscosity of biofluids and temperature at which the experiment is conducted. To perform fSPT measurements on F-pARG-HA NPs dispersed in $90 \%$ vol. biological fluids, nanoparticle formulations were first diluted 15 times in distilled water and $5 \mu \mathrm{l}$ of the prepared dilutions was then added to $45 \mu \mathrm{l}$ of distilled water or biofluids (e.g., human serum and ascites) and incubated for 1 hour at $37^{\circ} \mathrm{C}$. Afterwards, $7 \mu 1$ of each sample was mounted on a microscope slide in the middle of a secure-seal adhesive spacer ( 8 wells, $9 \mathrm{~mm}$ diameter, $0.12 \mathrm{~mm}$ deep, Invitrogen, Merelbeke, Belgium) and the slide was covered by a cover slip $(24 \times 50 \mathrm{~mm})$ in order to avoid sample evaporation and to afford free NP diffusion during fSPT measurements. The slide was then placed on the swept field confocal microscope and movies were recorded focused at $\sim 5 \mu \mathrm{m}$ above the bottom of the microscope slide. The fSPT videos of different nanoformulations were recorded at room temperature $\left(22.5^{\circ} \mathrm{C}\right)$ with the NIS Elements software (Nikon, Japan) driving the EMCCD camera and a sweptfield confocal microscope equipped with a CFI Plan Apo VC 100× NA 1.4 oil immersion objective lens (Nikon, Japan). Thereafter, videos were analyzed using in-house developed software, as explained previously ${ }^{45-46}$.

\subsection{Cell culture}

The human ovarian cancer cell line SKOV-3 was used for in vitro experiments. Cells were cultured in McCoy's 5A medium supplemented with 10\% Fetal Bovine Serum (FBS) and penicillin/streptomycin, and maintained in an incubator at $37{ }^{\circ} \mathrm{C}$, in a humidified atmosphere with $5 \% \mathrm{CO}_{2}$. Cells were cultured until the $80-90 \%$ confluency and then detached using $0.25 \%$ trypsin/EDTA.

\subsection{Cell viability assay}

SKOV-3 cells were seeded in a 24-well plate at a density of 50,000 cells/well and then cultivated at 37 ${ }^{\circ} \mathrm{C}$. After $24 \mathrm{~h}$, cells were incubated with Cis-pARG-NPs or free cisplatin at a final platinum (Pt) concentration ranging from 1-100 $\mu \mathrm{M}$ in each well containing Opti-MEM (final volume $500 \mu \mathrm{L}$ ). Cells 
in the control group were treated with blank pARG-HA NPs at equivalent polymer concentrations to Cis-pARG-HA NPs. Cells were then incubated with all nanoparticle formulations for $24 \mathrm{~h}$ at $37^{\circ} \mathrm{C}$, with $5 \% \mathrm{CO}_{2}$. At the end of treatments, cell viability was determined by MTT assay. Cells were rinsed with PBS and $100 \mu \mathrm{L}$ of MTT solution (5 mg/mL MTT in PBS) was added to each well containing $400 \mu \mathrm{L}$ of McCoy's 5A and incubated for $3 \mathrm{~h}$ at $37{ }^{\circ} \mathrm{C}$. Afterwards, $500 \mu \mathrm{L}$ of DMSO was added to each well to solubilize formazan crystals. The plate was then covered with aluminum foil and place on the orbital shaker for $30 \mathrm{~min}$ at $100 \mathrm{rpm}$. The absorbance of solubilized formazan was determined with a Wallac Envision ${ }^{\mathrm{TM}}$ multilabel reader (PerkinElmer, Zaventem, Belgium) at $590 \mathrm{~nm}$ wavelength with reference at $690 \mathrm{~nm}$. The measured absorbance was normalized to that of cells treated with Opti-MEM alone.

\subsection{In vitro cellular uptake of pARG-HA NPs in the presence of biofluids}

SKOV-3 cells were seeded in a 24-well plate (50,000 cells/well) and allowed to grow in an incubator at $37{ }^{\circ} \mathrm{C}$ for $24 \mathrm{~h}$. For uptake studies in biofluids, F-pARG-HA NPs were pre-incubated in Opti-MEM, human serum or ascetic fluid ( $90 \%$ vol. of biological fluids) for $1 \mathrm{~h}$ at $37{ }^{\circ} \mathrm{C}$. Following pre-incubation in aforementioned media, F-pARG-HA NPs were added to each well $\left(3.2 \times 10^{6}\right.$ nanoparticles/well $)$ containing Opti-MEM (final volume: $500 \mu \mathrm{L}$ ) and incubated for $4 \mathrm{~h} 37^{\circ} \mathrm{C}$. At the end of the incubation time, cells were washed twice with DPBS [-], trypsinized and analyzed using flow cytometry (FACS Calibur Flow Cytometer, BD Bioscience, USA).

\subsection{Competitive inhibition study}

SKOV-3 cells were seeded in a 24-well plate at the density of 50,000 cells/well and allowed to grow for $24 \mathrm{~h}$ at $37{ }^{\circ} \mathrm{C}$. F-pARG-HA NPs, dispersed in serum-free medium, were added to each well $\left(3.2 \times 10^{6}\right.$ nanoparticles/well) containing Opti-MEM (final volume: $500 \mu \mathrm{L}$ ), with or without HA $(10 \mathrm{mg} / \mathrm{mL}) .4$ $\mathrm{h}$ post-exposure of the cells to NPs, the cells were washed twice with PBS, detached using trypsin/EDTA and cellular uptake was analyzed by Cytoflex (Beckman Coulter, Suarlée, Belgium). For confocal microscopy, SKOV-3 cells (150,000 cells/dish) were plated in a $35 \mathrm{~mm}$ glass-bottomed petri dish (Greiner Bio-One, Frickenhausen, Germany) and incubated for $24 \mathrm{~h}$ at $37{ }^{\circ} \mathrm{C}$. Thereafter, cells were incubated with F-pARG-HA NPs suspended in Opti-MEM $\left(9.6 \times 10^{6}\right.$ nanoparticles/dish), either with or without HA $(10 \mathrm{mg} / \mathrm{mL}) .4 \mathrm{~h}$ upon incubation, cells were washed twice with PBS, fixed with paraformaldehyde (PFA) (4\% in PBS), and cell nuclei were stained with Hoechst $33342(1 \mu \mathrm{g} / \mathrm{mL})$. Cellular uptake was subsequently analyzed using confocal microscopy (C2 Nikon).

\subsection{In vivo therapeutic efficacy of Cis-pARG-HA NPs}

To establish a rat model of IP disseminated human ovarian cancer, $18 \times 10^{6}$ SKOV-3 Luc IP2 cells, suspended in $5 \mathrm{~mL}$ DPBS [-], were IP injected into athymic female nude rats (8-10 week old, $\geq 200 \mathrm{~g}$ ). Tumor growth was monitored using an IVIS Lumina II whole body imaging system (PerkinElmer, Zaventem, Belgium) until the bioluminescence signal reached $1 \times 10^{8}$ radians (photons $/ \mathrm{sec} / \mathrm{cm}^{2} / \mathrm{surface}$ area: 1-2 weeks). For bioluminescence imaging, in brief, rats were anesthetized by inhalation of $2 \%$ vol. isoflurane, and injected intraperitoneally with $1.5 \mathrm{~mL}$ of $15 \mathrm{mg} / \mathrm{mL}$ VivoGlo ${ }^{\mathrm{TM}}$ Luciferin (Promega, Madison, WI, USA) 10 min prior to imaging with identical system settings. Thereafter, tumor-bearing rats were randomly assigned to different treatments groups $(n=3-4)$ and dosed with free cisplatin (5 $\mathrm{mg} / \mathrm{kg}$ ) or Cis-pARG-HA NPs (at $5 \mathrm{mg} / \mathrm{kg}$ dose-equivalent level of platinum) through a single IP administration via IP injection or IP nebulization (i.e., PIPAC). Control animals were intraperitoneally injected with DPBS [-] alone.

The PIPAC procedure was performed as described in our previous study ${ }^{54}$. Briefly, a $5 \mathrm{~mm}$ and an 11 mm balloon safety trocar (Kii ${ }^{\circledR}$ Fios ${ }^{\circledR}$ First Entry, Applied Medical, Rancho, Santa Margarita, CA, USA) were inserted into the abdominal wall of rats (under continuous anesthesia by $4 \%$ vol. sevoflurane) placed in a supine position and fixed in a stable position at all four extremities. A constant $8 \mathrm{mmHg}$ 
carbon dioxide-based capnoperitoneum was established and maintained during the whole procedure. Next, a $5 \mathrm{~mm}$ camera (laparoscope) and a nebulizer (Micropump-MIP ${ }^{\circledR}$ ) (Reger Medizintechnik, Rottweil, Germany) were introduced to the $5 \mathrm{~mm}$ and $11 \mathrm{~mm}$ balloon trocars, respectively. The nebulizer was connected to an intravenous high pressure injector using a high pressure line and its nozzle orifice was secured just inside the peritoneal cavity. Thereafter, the high pressure injector was activated and a pressurized aerosol containing either free cisplatin of Cis-pARG-HA NPs in saline was applied to the peritoneal cavity at a flow rate of $0.5 \mathrm{~mL} / \mathrm{s}$ and a maximal upstream pressure of 20 bars. The constant capnoperitoneum of $8 \mathrm{mmHg}$ was maintained for $30 \mathrm{~min}$, and afterwards the aerosol was evacuated via a Closed Aerosol Waste system (CAWS). Finally, the trocars were retracted and the PIPAC procedure was terminated by suturing the laparoscopic incisions (Coated 4-0 VICRYL ${ }^{\mathrm{TM}}$ Plus ETHICON ${ }^{\circledR}$, Johnson and Johnson International, Diegem, Belgium) and intramuscular administration of an analgesic (Ketoprofen, Sanofi, Diegem, Belgium).

To evaluate the antitumor activity of each IP therapeutic approach, tumor growth and progression of metastasis were monitored via serial bioluminescence imaging for 3 weeks post-treatment. During the study period, animals were observed every 2 days for weight change and signs of pain. Rats showing the gross sign of toxicity, $>20 \%$ loss in the body weight or bloated abdomen, were euthanized according to an approved animal procedure. 21 days after treatments at the latest, the animals were sacrificed and all peritoneal nodules and other organs in the peritoneal cavity were carefully examined and excised. The extent of tumor dissemination, weight of tumor nodules and overall survival were subsequently recorded for each treatment group. Upon completion of in vivo imaging, the intensity of bioluminescence signals emanating from tumor growth were quantified by Living Image Software. Tumor growth was determined by comparing the changes in bioluminescence signal intensities to the baseline. These changes were quantified via gating on the area of tumor growth in the peritoneal cavity and measured by an average photon flux in radians (photons $/ \mathrm{s} / \mathrm{cm}^{2} /$ surface area), which eventually allowed normalization of different signal intensities between rats in all treatments groups and also in the same rat over time.

\subsection{In vivo biodistribution studies}

SKOV-3 Luc IP2 cells $\left(18 \times 10^{6}\right.$ cells suspended in $5 \mathrm{~mL}$ DPBS [-]) were IP injected into athymic female nude rats (8-10 weeks old, $\geq 200 \mathrm{~g}$ ) and allowed to grow until the bioluminescence signal intensity from tumor growth reached $1 \times 10^{8}$ radians (photons $/ \mathrm{sec} / \mathrm{cm}^{2} /$ surface area: $\sim 1$-2 weeks). Afterwards, rats bearing IP disseminated tumors were randomly divided into 2 groups $(n=3)$ which received IP administration of F-pARG-HA NPs $\left(5 \times 10^{9}\right.$ nanoparticles/rat $)$ either via injection $(5 \mathrm{~mL})$ or nebulization $(10 \mathrm{~mL}$, resulting in the peritoneal administration of $5 \mathrm{ml}$ when taking into account the dead volume). Rats in the control group were intraperitoneally injected with DPBS [-] alone. $48 \mathrm{~h}$ postIP administration, all animals were sacrificed and peritoneal nodules and other organs were harvested, fixed in $4 \%$ paraformaldehyde in PBS (pH:7.4), snap-frozen in liquid nitrogen and stored at $-80{ }^{\circ} \mathrm{C}$ for further analysis. Thereafter, all samples were cryosectioned at a thickness of $10 \mu \mathrm{m}$ and cryosections were equilibrated at RT, washed with DPBS[-] and nuclei of cells were counterstained with Hoechst $33342(10 \mu \mathrm{g} / \mathrm{mL})$. The stained tissue sections were subsequently assessed by fluorescence confocal microscopy (C2 Nikon) and images were processed and analyzed by Image J.

\subsection{Statistical analysis}

Results were analyzed using GraphPad Prism 7 software and statistical comparisons were conducted with one-way or two-way ANOVA. A P-value $<0.05$ was considered statistically significant. Data were expressed as mean \pm standard deviation (SD). 


\section{Acknowledgement}

This work was financially supported by the European Union's Horizon 2020 research and innovation program Marie Sklodowska-Curie Innovative Training Networks (ITN) under grant No. 676137, the research foundation Flanders (Research grant G006714N \& G061119N) and Ghent University (BOF GOA).

\section{References}

(1) Reid, B. M.; Permuth, J. B.; Sellers, T. A. Epidemiology of ovarian cancer: a review. Cancer Biol Med 2017, 14 (1), 9-32, DOI: 10.20892/j.issn.2095-3941.2016.0084.

(2) Coccolini, F.; Gheza, F.; Lotti, M.; Virzi, S.; Iusco, D.; Ghermandi, C.; Melotti, R.; Baiocchi, G.; Giulini, S. M.; Ansaloni, L.; Catena, F. Peritoneal carcinomatosis. World J Gastroenterol 2013, 19 (41), 6979-6994, DOI: 10.3748/wjg.v19.i41.6979.

(3) Banerjee, S.; Kaye, S. B. New strategies in the treatment of ovarian cancer: current clinical perspectives and future potential. Clin Cancer Res 2013, 19 (5), 961-968, DOI: 10.1158/10780432.CCR-12-2243.

(4) Tempfer, C. B. Pressurized intraperitoneal aerosol chemotherapy as an innovative approach to treat peritoneal carcinomatosis. Med Hypotheses 2015, 85 (4), 480-484, DOI: 10.1016/j.mehy.2015.07.001.

(5) Dedrick, R. L.; Myers, C. E.; Bungay, P. M.; DeVita, V. T., Jr. Pharmacokinetic rationale for peritoneal drug administration in the treatment of ovarian cancer. Cancer Treat Rep 1978, 62 (1), 1-11.

(6) Dedrick, R. L. Theoretical and experimental bases of intraperitoneal chemotherapy. Semin Oncol 1985, 12 (3 Suppl 4), 1-6.

(7) Los, G.; Mutsaers, P. H.; van der Vijgh, W. J.; Baldew, G. S.; de Graaf, P. W.; McVie, J. G. Direct diffusion of cis-diamminedichloroplatinum(II) in intraperitoneal rat tumors after intraperitoneal chemotherapy: a comparison with systemic chemotherapy. Cancer Res 1989, 49 (12), 3380-3384.

(8) Dehal, A.; Smith, J. J.; Nash, G. M. Cytoreductive surgery and intraperitoneal chemotherapy: an evidence-based review-past, present and future. J Gastrointest Oncol 2016, 7 (1), 143-157, DOI: 10.3978/j.issn.2078-6891.2015.112.

(9) Baratti, D.; Kusamura, S.; Pietrantonio, F.; Guaglio, M.; Niger, M.; Deraco, M. Progress in treatments for colorectal cancer peritoneal metastases during the years 2010-2015. A systematic review. Crit Rev Oncol Hematol 2016, 100, 209-222, DOI: 10.1016/j.critrevonc.2016.01.017.

(10) Girshally, R.; Demtroder, C.; Albayrak, N.; Zieren, J.; Tempfer, C.; Reymond, M. A. Pressurized intraperitoneal aerosol chemotherapy (PIPAC) as a neoadjuvant therapy before cytoreductive surgery and hyperthermic intraperitoneal chemotherapy. World J Surg Oncol 2016, 14 (1), 253, DOI: 10.1186/s12957-016-1008-0.

(11) Solass, W.; Kerb, R.; Murdter, T.; Giger-Pabst, U.; Strumberg, D.; Tempfer, C.; Zieren, J.; Schwab, M.; Reymond, M. A. Intraperitoneal chemotherapy of peritoneal carcinomatosis using pressurized aerosol as an alternative to liquid solution: first evidence for efficacy. Ann Surg Oncol 2014, 21 (2), 553-559, DOI: 10.1245/s10434-013-3213-1.

(12) Solass, W.; Hetzel, A.; Nadiradze, G.; Sagynaliev, E.; Reymond, M. A. Description of a novel approach for intraperitoneal drug delivery and the related device. Surg Endosc 2012, 26 (7), 1849-1855, DOI: $10.1007 / \mathrm{s} 00464-012-2148-0$.

(13) Solass, W.; Herbette, A.; Schwarz, T.; Hetzel, A.; Sun, J. S.; Dutreix, M.; Reymond, M. A. Therapeutic approach of human peritoneal carcinomatosis with Dbait in combination with capnoperitoneum: proof of concept. Surg Endosc 2012, 26 (3), 847-852, DOI: 10.1007/s00464-0111964-y.

(14) Dedrick, R. L.; Flessner, M. F. Pharmacokinetic problems in peritoneal drug administration: tissue penetration and surface exposure. J Natl Cancer Inst 1997, 89 (7), 480-487, DOI: 10.1093/jnci/89.7.480.

(15) Wolinsky, J. B.; Colson, Y. L.; Grinstaff, M. W. Local drug delivery strategies for cancer treatment: gels, nanoparticles, polymeric films, rods, and wafers. J Control Release 2012, 159 (1), 14-26, DOI: 10.1016/j.jconrel.2011.11.031. 
(16) Dakwar, G. R.; Shariati, M.; Willaert, W.; Ceelen, W.; De Smedt, S. C.; Remaut, K. Nanomedicinebased intraperitoneal therapy for the treatment of peritoneal carcinomatosis - Mission possible? Adv Drug Deliv Rev 2017, 108, 13-24, DOI: 10.1016/j.addr.2016.07.001.

(17) Nowacki, M.; Peterson, M.; Kloskowski, T.; McCabe, E.; Guiral, D. C.; Polom, K.; Pietkun, K.; Zegarska, B.; Pokrywczynska, M.; Drewa, T.; Roviello, F.; Medina, E. A.; Habib, S. L.; Zegarski, W. Nanoparticle as a novel tool in hyperthermic intraperitoneal and pressurized intraperitoneal aerosol chemotheprapy to treat patients with peritoneal carcinomatosis. Oncotarget 2017, 8 (44), 78208-78224, DOI: 10.18632/oncotarget.20596.

(18) Platt, V. M.; Szoka, F. C., Jr. Anticancer therapeutics: targeting macromolecules and nanocarriers to hyaluronan or CD44, a hyaluronan receptor. Mol Pharm 2008, 5 (4), 474-486, DOI: $10.1021 / \mathrm{mp} 800024 \mathrm{~g}$.

(19) Lee, H.; Lee, K.; Kim, I. K.; Park, T. G. Synthesis, characterization, and in vivo diagnostic applications of hyaluronic acid immobilized gold nanoprobes. Biomaterials 2008, 29 (35), 4709-4718, DOI: 10.1016/j.biomaterials.2008.08.038.

(20) Mironov, V.; Kasyanov, V.; Zheng Shu, X.; Eisenberg, C.; Eisenberg, L.; Gonda, S.; Trusk, T.; Markwald, R. R.; Prestwich, G. D. Fabrication of tubular tissue constructs by centrifugal casting of cells suspended in an in situ crosslinkable hyaluronan-gelatin hydrogel. Biomaterials 2005, 26 (36), 76287635, DOI: 10.1016/j.biomaterials.2005.05.061.

(21) Oh, E. J.; Park, K.; Kim, K. S.; Kim, J.; Yang, J. A.; Kong, J. H.; Lee, M. Y.; Hoffman, A. S.; Hahn, S. K. Target specific and long-acting delivery of protein, peptide, and nucleotide therapeutics using hyaluronic acid derivatives. $J$ Control Release 2010, 141 (1), 2-12, DOI: 10.1016/j.jconrel.2009.09.010.

(22) Toole, B. P. Hyaluronan: from extracellular glue to pericellular cue. Nat Rev Cancer 2004, 4 (7), 528-539, DOI: 10.1038/nrc1391.

(23) Shah, V.; Taratula, O.; Garbuzenko, O. B.; Taratula, O. R.; Rodriguez-Rodriguez, L.; Minko, T. Targeted nanomedicine for suppression of CD44 and simultaneous cell death induction in ovarian cancer: an optimal delivery of siRNA and anticancer drug. Clin Cancer Res 2013, 19 (22), 6193-6204, DOI: 10.1158/1078-0432.CCR-13-1536.

(24) Choi, K. Y.; Chung, H.; Min, K. H.; Yoon, H. Y.; Kim, K.; Park, J. H.; Kwon, I. C.; Jeong, S. Y. Self-assembled hyaluronic acid nanoparticles for active tumor targeting. Biomaterials 2010, 31 (1), 106114, DOI: 10.1016/j.biomaterials.2009.09.030.

(25) Bae, K. H.; Tan, S.; Yamashita, A.; Ang, W. X.; Gao, S. J.; Wang, S.; Chung, J. E.; Kurisawa, M. Hyaluronic acid-green tea catechin micellar nanocomplexes: Fail-safe cisplatin nanomedicine for the treatment of ovarian cancer without off-target toxicity. Biomaterials 2017, 148, 41-53, DOI: 10.1016/j.biomaterials.2017.09.027.

(26) Oyarzun-Ampuero, F. A.; Goycoolea, F. M.; Torres, D.; Alonso, M. J. A new drug nanocarrier consisting of polyarginine and hyaluronic acid. Eur J Pharm Biopharm 2011, 79 (1), 54-57, DOI: 10.1016/j.ejpb.2011.04.008.

(27) Carton, F.; Chevalier, Y.; Nicoletti, L.; Tarnowska, M.; Stella, B.; Arpicco, S.; Malatesta, M.; Jordheim, L. P.; Briancon, S.; Lollo, G. Rationally designed hyaluronic acid-based nano-complexes for pentamidine delivery. Int J Pharm 2019, 568, 118526, DOI: 10.1016/j.ijpharm.2019.118526.

(28) Lollo, G.; Benoit, J. P.; Brachet, M. Drug delivery system, European Patent Office (EP18306201.7). 2018.

(29) Matha, K.; Lollo, G.; Taurino, G.; Respaud, R.; Marigo, I.; Shariati, M.; Bussolati, O.; Vermeulen, A.; Remaut, K.; Benoit, J. P. Bioinspired hyaluronic acid and polyarginine nanoparticles for DACHPt delivery. Eur J Pharm Biopharm 2020, 150, 1-13, DOI: 10.1016/j.ejpb.2020.02.008.

(30) Rosenberg, B.; VanCamp, L.; Trosko, J. E.; Mansour, V. H. Platinum compounds: a new class of potent antitumour agents. Nature 1969, 222 (5191), 385-386, DOI: 10.1038/222385a0.

(31) Lebwohl, D.; Canetta, R. Clinical development of platinum complexes in cancer therapy: an historical perspective and an update. Eur J Cancer 1998, 34 (10), 1522-1534, DOI: 10.1016/s09598049(98)00224-x.

(32) Kelland, L. The resurgence of platinum-based cancer chemotherapy. Nat Rev Cancer 2007, 7 (8), 573-584, DOI: $10.1038 / n r c 2167$. 
(33) Oberoi, H. S.; Nukolova, N. V.; Kabanov, A. V.; Bronich, T. K. Nanocarriers for delivery of platinum anticancer drugs. Adv Drug Deliv Rev 2013, 65 (13-14), 1667-1685, DOI: 10.1016/j.addr.2013.09.014.

(34) Kartalou, M.; Essigmann, J. M. Mechanisms of resistance to cisplatin. Mutat Res 2001, 478 (1-2), 23-43, DOI: 10.1016/s0027-5107(01)00141-5.

(35) Cho, E. J.; Sun, B.; Doh, K. O.; Wilson, E. M.; Torregrosa-Allen, S.; Elzey, B. D.; Yeo, Y. Intraperitoneal delivery of platinum with in-situ crosslinkable hyaluronic acid gel for local therapy of ovarian cancer. Biomaterials 2015, 37, 312-319, DOI: 10.1016/j.biomaterials.2014.10.039.

(36) Mochida, Y.; Cabral, H.; Kataoka, K. Polymeric micelles for targeted tumor therapy of platinum anticancer drugs. Expert Opin Drug Deliv 2017, 14 (12), 1423-1438, DOI: 10.1080/17425247.2017.1307338.

(37) Nishiyama, N.; Okazaki, S.; Cabral, H.; Miyamoto, M.; Kato, Y.; Sugiyama, Y.; Nishio, K.; Matsumura, Y.; Kataoka, K. Novel cisplatin-incorporated polymeric micelles can eradicate solid tumors in mice. Cancer Res 2003, 63 (24), 8977-8983.

(38) Jeong, Y. I.; Kim, S. T.; Jin, S. G.; Ryu, H. H.; Jin, Y. H.; Jung, T. Y.; Kim, I. Y.; Jung, S. Cisplatinincorporated hyaluronic acid nanoparticles based on ion-complex formation. J Pharm Sci 2008, 97 (3), 1268-1276, DOI: 10.1002/jps.21103.

(39) Huang, Y. Q.; Zhang, R.; Zhao, Y. K.; Chen, H.; Jiang, R. C.; Liu, X. F.; Fan, Q. L.; Wang, L. H.; Huang, W. Self-assembled nanoparticles based on a cationic conjugated polymer/hyaluronan-cisplatin complex as a multifunctional platform for simultaneous tumor-targeting cell imaging and drug delivery. New J Chem 2017, 41 (12), 4998-5006, DOI: 10.1039/c6nj04128k.

(40) Surnar, B.; Sharma, K.; Jayakannan, M. Core-shell polymer nanoparticles for prevention of GSH drug detoxification and cisplatin delivery to breast cancer cells. Nanoscale 2015, 7 (42), 17964-17979, DOI: $10.1039 / c 5 n r 04963 f$.

(41) Johnstone, T. C.; Suntharalingam, K.; Lippard, S. J. The Next Generation of Platinum Drugs: Targeted Pt(II) Agents, Nanoparticle Delivery, and Pt(IV) Prodrugs. Chem Rev 2016, 116 (5), 34363486, DOI: 10.1021/acs.chemrev.5b00597.

(42) Xiao, H. H.; Yan, L. S.; Dempsey, E. M.; Song, W. T.; Qi, R. G.; Li, W. L.; Huang, Y. B.; Jing, X. B.; Zhou, D. F.; Ding, J. X.; Chen, X. S. Recent progress in polymer-based platinum drug delivery systems. Prog Polym Sci 2018, 87, 70-106, DOI: 10.1016/j.progpolymsci.2018.07.004.

(43) Monopoli, M. P.; Aberg, C.; Salvati, A.; Dawson, K. A. Biomolecular coronas provide the biological identity of nanosized materials. Nat Nanotechnol 2012, 7 (12), 779-786, DOI: 10.1038/nnano.2012.207.

(44) Mahmoudi, M.; Lynch, I.; Ejtehadi, M. R.; Monopoli, M. P.; Bombelli, F. B.; Laurent, S. Proteinnanoparticle interactions: opportunities and challenges. Chem Rev 2011, 111 (9), 5610-5637, DOI: $10.1021 / \mathrm{cr} 100440 \mathrm{~g}$.

(45) Dakwar, G. R.; Zagato, E.; Delanghe, J.; Hobel, S.; Aigner, A.; Denys, H.; Braeckmans, K.; Ceelen, W.; De Smedt, S. C.; Remaut, K. Colloidal stability of nano-sized particles in the peritoneal fluid: towards optimizing drug delivery systems for intraperitoneal therapy. Acta Biomater 2014, 10 (7), 2965 2975, DOI: 10.1016/j.actbio.2014.03.012.

(46) Dakwar, G. R.; Braeckmans, K.; Demeester, J.; Ceelen, W.; De Smedt, S. C.; Remaut, K. Disregarded Effect of Biological Fluids in siRNA Delivery: Human Ascites Fluid Severely Restricts Cellular Uptake of Nanoparticles. ACS Appl Mater Interfaces 2015, 7 (43), 24322-24329, DOI: 10.1021/acsami.5b08805.

(47) Cherukula, K.; Bae, W. K.; Lee, J. H.; Park, I. K. Programmed 'triple-mode' anti-tumor therapy: Improving peritoneal retention, tumor penetration and activatable drug release properties for effective inhibition of peritoneal carcinomatosis. Biomaterials 2018, 169, 45-60, DOI: 10.1016/j.biomaterials.2018.03.051.

(48) Gao, Y.; Foster, R.; Yang, X.; Feng, Y.; Shen, J. K.; Mankin, H. J.; Hornicek, F. J.; Amiji, M. M.; Duan, Z. Up-regulation of CD44 in the development of metastasis, recurrence and drug resistance of ovarian cancer. Oncotarget 2015, 6 (11), 9313-9326, DOI: 10.18632/oncotarget.3220.

(49) De Stefano, I.; Battaglia, A.; Zannoni, G. F.; Prisco, M. G.; Fattorossi, A.; Travaglia, D.; Baroni, S.; Renier, D.; Scambia, G.; Ferlini, C.; Gallo, D. Hyaluronic acid-paclitaxel: effects of intraperitoneal administration against CD44(+) human ovarian cancer xenografts. Cancer Chemother Pharmacol 2011, 68 (1), 107-116, DOI: 10.1007/s00280-010-1462-2. 
(50) Picaud, L.; Thibault, B.; Mery, E.; Ouali, M.; Martinez, A.; Delord, J. P.; Couderc, B.; Ferron, G. Evaluation of the effects of hyaluronic acid-carboxymethyl cellulose barrier on ovarian tumor progression. J Ovarian Res 2014, 7, 40, DOI: 10.1186/1757-2215-7-40.

(51) Ceelen, W. P.; Flessner, M. F. Intraperitoneal therapy for peritoneal tumors: biophysics and clinical evidence. Nat Rev Clin Oncol 2010, 7 (2), 108-115, DOI: 10.1038/nrclinonc.2009.217.

(52) Tempfer, C.; Giger-Pabst, U.; Hilal, Z.; Dogan, A.; Rezniczek, G. A. Pressurized intraperitoneal aerosol chemotherapy (PIPAC) for peritoneal carcinomatosis: systematic review of clinical and experimental evidence with special emphasis on ovarian cancer. Arch Gynecol Obstet 2018, 298 (2), 243-257, DOI: 10.1007/s00404-018-4784-7.

(53) Dueckelmann, A. M.; Fink, D.; Harter, P.; Heinzelmann, V.; Marth, C.; Mueller, M.; Reinthaller, A.; Tamussino, K.; Wimberger, P.; Sehouli, J. The use of PIPAC (pressurized intraperitoneal aerosol chemotherapy) in gynecological oncology: a statement by the "Arbeitsgemeinschaft Gynaekologische Onkologie Studiengruppe Ovarialkarzinom (AGO-OVAR)", the Swiss and Austrian AGO, and the North-Eastern German Society of Gynaecologic Oncology. Arch Gynecol Obstet 2018, 297 (4), 837846, DOI: 10.1007/s00404-018-4673-0.

(54) Shariati, M.; Zhang, H.; Van de Sande, L.; Descamps, B.; Vanhove, C.; Willaert, W.; Ceelen, W.; De Smedt, S. C.; Remaut, K. High Pressure Nebulization (PIPAC) Versus Injection for the Intraperitoneal Administration of mRNA Complexes. Pharm Res 2019, 36 (9), 126, DOI: 10.1007/s11095-019-2646-z.

(55) Van De Sande, L.; Graversen, M.; Hubner, M.; Pocard, M.; Reymond, M.; Vaira, M.; Cosyns, S.; Willaert, W.; Ceelen, W. Intraperitoneal aerosolization of albumin-stabilized paclitaxel nanoparticles (Abraxane) for peritoneal carcinomatosis - a phase I first-in-human study. Pleura Peritoneum 2018, 3 (2), 20180112, DOI: 10.1515/pp-2018-0112.

(56) Flessner, M. F.; Choi, J.; He, Z.; Credit, K. Physiological characterization of human ovarian cancer cells in a rat model of intraperitoneal antineoplastic therapy. J Appl Physiol (1985) 2004, 97 (4), 15185126, DOI: 10.1152/japplphysiol.00305.2004.

(57) Van de Sande, L.; Willaert, W.; Cosyns, S.; De Clercq, K.; Shariati, M.; Remaut, K.; Ceelen, W. Establishment of a rat ovarian peritoneal metastasis model to study pressurized intraperitoneal aerosol chemotherapy (PIPAC). BMC Cancer 2019, 19 (1), 424, DOI: 10.1186/s12885-019-5658-5.

(58) Van de Sande, L.; Rahimi-Gorgi, M.; Giordano, S.; Matteo, C.; Davoli, D.; Detlefsen, S.; D’Herde, K.; Coetsier, M.; Braet, H.; Shariati, M.; Remaut, K.; Xie, F.; Cosyns, S.; Debbaut, C.; Ghorbaniasl, G.; Willaert, W.; Ceelen, W. Electrostatic intraperitoneal aerosol delivery of nanoparticles: proof of concept and preclinical validation Adv Healthc Mater 2020, In Press.

(59) Gohler, D.; Khosrawipour, V.; Khosrawipour, T.; Diaz-Carballo, D.; Falkenstein, T. A.; Zieren, J.; Stintz, M.; Giger-Pabst, U. Technical description of the microinjection pump (MIP((R))) and granulometric characterization of the aerosol applied for pressurized intraperitoneal aerosol chemotherapy (PIPAC). Surg Endosc 2017, 31 (4), 1778-1784, DOI: 10.1007/s00464-016-5174-5.

(60) Khosrawipour, V.; Khosrawipour, T.; Diaz-Carballo, D.; Forster, E.; Zieren, J.; Giger-Pabst, U. Exploring the Spatial Drug Distribution Pattern of Pressurized Intraperitoneal Aerosol Chemotherapy (PIPAC). Ann Surg Oncol 2016, 23 (4), 1220-1224, DOI: 10.1245/s10434-015-4954-9.

(61) Khosrawipour, V.; Khosrawipour, T.; Kern, A. J.; Osma, A.; Kabakci, B.; Diaz-Carballo, D.; Forster, E.; Zieren, J.; Fakhrian, K. Distribution pattern and penetration depth of doxorubicin after pressurized intraperitoneal aerosol chemotherapy (PIPAC) in a postmortem swine model. $J$ Cancer Res Clin Oncol 2016, 142 (11), 2275-2280, DOI: 10.1007/s00432-016-2234-0.

(62) Bellendorf, A.; Khosrawipour, V.; Khosrawipour, T.; Siebigteroth, S.; Cohnen, J.; Diaz-Carballo, D.; Bockisch, A.; Zieren, J.; Giger-Pabst, U. Scintigraphic peritoneography reveals a non-uniform (99m)Tc-Pertechnetat aerosol distribution pattern for Pressurized Intra-Peritoneal Aerosol Chemotherapy (PIPAC) in a swine model. Surg Endosc 2018, 32 (1), 166-174, DOI: 10.1007/s00464017-5652-4.

(63) Khosrawipour, V.; Khosrawipour, T.; Falkenstein, T. A.; Diaz-Carballo, D.; Forster, E.; Osma, A.; Adamietz, I. A.; Zieren, J.; Fakhrian, K. Evaluating the Effect of Micropump(c) Position, Internal Pressure and Doxorubicin Dosage on Efficacy of Pressurized Intra-peritoneal Aerosol Chemotherapy (PIPAC) in an Ex Vivo Model. Anticancer Res 2016, 36 (9), 4595-4600, DOI: 10.21873/anticanres. 11008 
(64) Gohler, D.; Grosse, S.; Bellendorf, A.; Falkenstein, T. A.; Ouaissi, M.; Zieren, J.; Stintz, M.; GigerPabst, U. Hyperthermic intracavitary nanoaerosol therapy (HINAT) as an improved approach for pressurised intraperitoneal aerosol chemotherapy (PIPAC): Technical description, experimental validation and first proof of concept. Beilstein $J$ Nanotechnol 2017, 8, 2729-2740, DOI: 10.3762/bjnano.8.272.

(65) Reymond, M.; Demtroeder, C.; Solass, W.; Winnekendonk, G.; Tempfer, C. Electrostatic precipitation Pressurized IntraPeritoneal Aerosol Chemotherapy (ePIPAC): first in-human application. Pleura Peritoneum 2016, 1 (2), 109-116, DOI: 10.1515/pp-2016-0005.

(66) Braeckmans, K.; Buyens, K.; Bouquet, W.; Vervaet, C.; Joye, P.; De Vos, F.; Plawinski, L.; Doeuvre, L.; Angles-Cano, E.; Sanders, N. N.; Demeester, J.; De Smedt, S. C. Sizing nanomatter in biological fluids by fluorescence single particle tracking. Nano Lett 2010, 10 (11), 4435-4442, DOI: $10.1021 / \mathrm{nl} 103264 \mathrm{u}$.

\section{Table of Content Graphic}
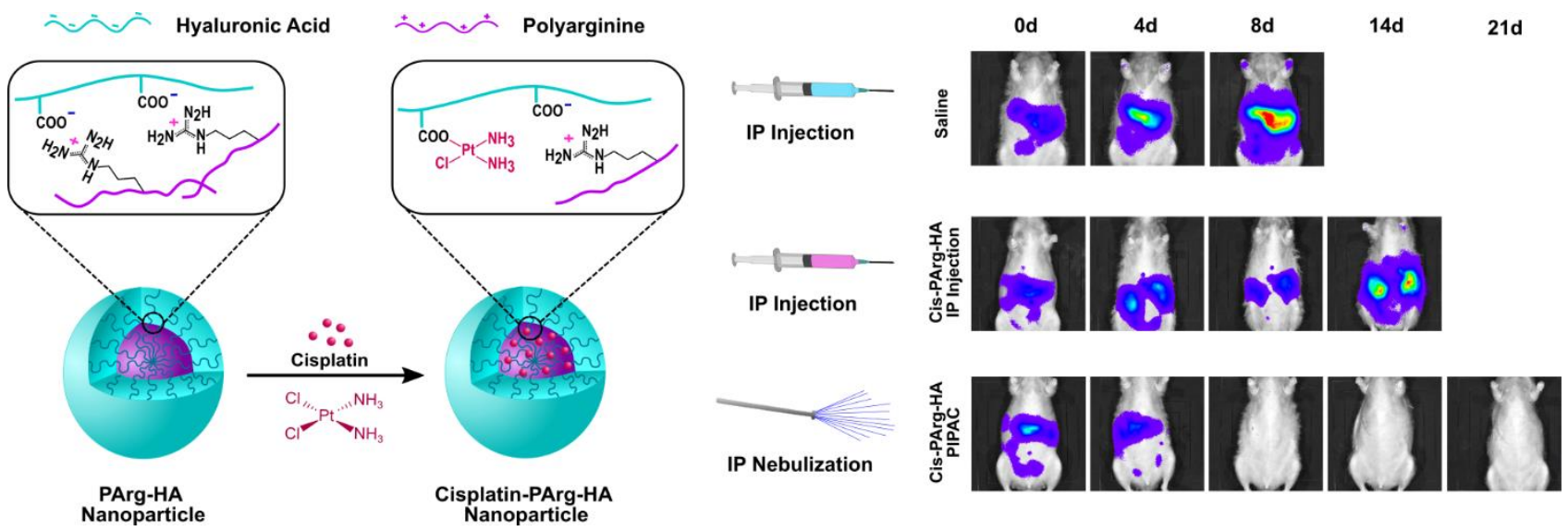